\title{
Three-dimensional simulations of plasma turbulence in the RFX-mod scrape-off layer and comparison with experimental measurements
}

Fabio Riva, Nicola Vianello, Monica Spolaore, Paolo Ricci, Roberto Cavazzana, Lionello Marrelli, and Silvia Spagnolo

Citation: Physics of Plasmas 25, 022305 (2018); doi: 10.1063/1.5008803

View online: https://doi.org/10.1063/1.5008803

View Table of Contents: http://aip.scitation.org/toc/php/25/2

Published by the American Institute of Physics

\section{Articles you may be interested in}

Poloidal asymmetries of flows in the Tore Supra tokamak

Physics of Plasmas 25, 020704 (2018); 10.1063/1.5022122

From single helical relaxed states to helical equilibria

Physics of Plasmas 25, 022112 (2018); 10.1063/1.5019646

Global 3D two-fluid simulations of the tokamak edge region: Turbulence, transport, profile evolution, and spontaneous $\mathrm{E} \times \mathrm{B}$ rotation

Physics of Plasmas 24, 055903 (2017); 10.1063/1.4978885

Observation of trapped-electron-mode microturbulence in reversed field pinch plasmas

Physics of Plasmas 25, 010701 (2018); 10.1063/1.5010198

Core radial electric field and transport in Wendelstein 7-X plasmas

Physics of Plasmas 25, 022508 (2018); 10.1063/1.4999842

Multi-scale transport in the DIII-D ITER baseline scenario with direct electron heating and projection to ITER

Physics of Plasmas 25, 022509 (2018); 10.1063/1.5011387

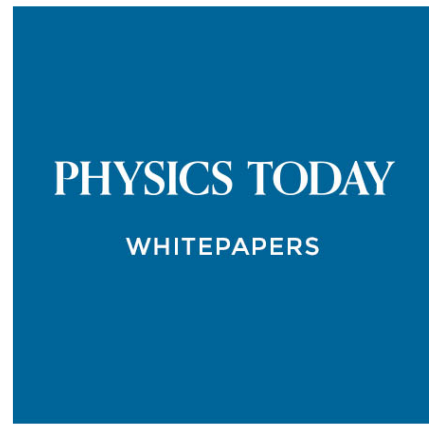

ADVANCES IN PRECISION MOTION CONTROL

Piezo Flexure Mechanisms and Air Bearings
READ NOW

PRESENTED BY

PI 


\title{
Three-dimensional simulations of plasma turbulence in the RFX-mod scrape-off layer and comparison with experimental measurements
}

\author{
Fabio Riva, ${ }^{1, a)}$ Nicola Vianello, ${ }^{2}$ Monica Spolaore, ${ }^{2}$ Paolo Ricci, ${ }^{1}$ Roberto Cavazzana, ${ }^{2}$ \\ Lionello Marrelli, ${ }^{2}$ and Silvia Spagnolo ${ }^{2}$ \\ ${ }^{1}$ École Polytechnique Fédérale de Lausanne (EPFL), Swiss Plasma Center (SPC), CH-1015 Lausanne, \\ Switzerland \\ ${ }^{2}$ Consorzio RFX (CNR, ENEA, INFN, Università di Padova, Acciaierie Venete SpA) Corso Stati \\ Uniti 4 - 35127 Padova, Italy
}

(Received 10 October 2017; accepted 2 February 2018; published online 26 February 2018)

The tokamak scrape-off layer (SOL) plasma dynamics is investigated in a circular limiter configuration with a low edge safety factor. Focusing on the experimental parameters of two ohmic tokamak inner-wall limited plasma discharges in RFX-mod [Sonato et al., Fusion Eng. Des. 74, 97 (2005)], nonlinear SOL plasma simulations are performed with the GBS code [Ricci et al., Plasma Phys. Controlled Fusion 54, 124047 (2012)]. The numerical results are compared with the experimental measurements, assessing the reliability of the GBS model in describing the RFX-mod SOL plasma dynamics. It is found that the simulations are able to quantitatively reproduce the RFX-mod experimental measurements of the electron plasma density, electron temperature, and ion saturation current density $\left(j_{\text {sat }}\right)$ equilibrium profiles. Moreover, there are indications that the turbulent transport is driven by the same instability in the simulations and in the experiment, with coherent structures having similar statistical properties. On the other hand, it is found that the simulation results are not able to correctly reproduce the floating potential equilibrium profile and the $j_{\text {sat }}$ fluctuation level. It is likely that these discrepancies are, at least in part, related to simulating only the tokamak SOL region, without including the plasma dynamics inside the last close flux surface, and to the limits of applicability of the drift approximation. The turbulence drive is then identified from the nonlinear simulations and with the linear theory. It results that the inertial drift wave is the instability driving most of the turbulent transport in the considered discharges.

https://doi.org/10.1063/1.5008803

\section{INTRODUCTION}

Understanding the plasma dynamics in the tokamak scrape-off layer (SOL) is of crucial importance, since phenomena taking place in this region play a fundamental role in determining the overall performance of fusion devices. In fact, the SOL sets the boundary conditions for the tokamak core, it controls the impurity dynamics and the recycling level, and it is responsible for exhausting the tokamak power, thus determining the heat load at the vessel walls. ${ }^{1}$

Due to the complex nonlinear phenomena taking place in the SOL, the plasma dynamics in this region is usually investigated numerically, thanks to state-of-the-art simulation codes (see, e.g., Refs. 2-5). In the present paper, we focus on the tokamak limited SOL configuration. Besides being of interest as a stepping stone towards the simulation of more complex experimental scenarios, this configuration has recently attracted large attention since the ITER ${ }^{6}$ start-up and ramp-down phases will be performed using the highfield side part of the vacuum vessel as the limiting surface. ${ }^{7,8}$

In the past, extensive theoretical and numerical studies of the instabilities driving the SOL dynamics were performed (see, e.g., Refs. 9-12). It was found that, in the limited configuration, SOL turbulence is generally driven by drift-waves (DWs) and ballooning modes (BMs). ${ }^{12,13}$ It was

\footnotetext{
${ }^{a)}$ Electronic mail: fabio.riva@ukaea.uk
}

also demonstrated that these linear instabilities typically saturate due to a nonlinear local flattening of the plasma gradient and the resulting removal of the instability drive. ${ }^{14}$ These theoretical findings were subsequently validated against experimental measurements taken on a number of tokamaks around the world, such as TCV, MAST, EAST, Alcator CMod, ISTTOK, and Tore Supra, showing good agreement between simulations and experimental measurements of plasma turbulence. ${ }^{15-21}$ Moreover, using these observations and assuming that resistive BMs (RBMs) drive the SOL turbulence dynamics and that the parallel losses at the vessel are balanced by the turbulent transport, an analytical scaling for the equilibrium pressure gradient length was derived. ${ }^{22,23}$ It was found that this scaling is consistent with measurements taken on a number of experimental devices. ${ }^{24}$

The goal of the present paper is to investigate a SOL parameter regime that was not explored earlier and, in general, difficult to access experimentally. More precisely, we investigate the SOL plasma dynamics in a circular limiter configuration with a low safety factor at the last close flux surface (LCFS), $q_{L C F S} \lesssim 3$, for which the SOL turbulence is expected to be clearly in the inertial DW (InDW) regime. ${ }^{12}$ Our study is based on performing SOL turbulence simulations considering two tokamak circular plasma discharges carried out in the RFX-mod experiment ${ }^{25}$ with $q_{\mathrm{LCFS}} \approx 2,3$. The RFX-mod device can access such low safety factors thanks to an advanced feedback magnetic boundary control 
system, which allows stabilizing resistive wall modes and performing plasma discharges with $q_{L C F S} \approx 2$ without disruptions. ${ }^{26}$ We then carefully compare the simulation results with RFX-mod experimental measurements and we analyze the nature of the turbulence in the SOL of RFX-mod.

The present paper is organized as follows: In Sec. II, we discuss the RFX-mod experimental setup. Then, in Sec. III, we describe the simulations of the RFX-mod plasma discharges. In Sec. IV, the numerical results are validated against experimental measurements. The instability that drives the SOL plasma dynamics is identified thanks to nonlinear simulations and linear theory in Sec. V. Finally, we report our conclusions in Sec. VI. We note that the present paper is an extended version of the work illustrated in Chapter 6 of Ref. 27.

\section{THE EXPERIMENTAL SETUP}

The RFX-mod experiment is a flexible toroidal device with major radius $R=2 \mathrm{~m}$ and minor radius $a=0.459 \mathrm{~m}$, equipped with a set of 192 actively controlled coils that cover the whole vacuum vessel. ${ }^{25}$ While RFX-mod plasma discharges have been performed mainly in the reversed field pinch (RFP) configuration, recent developments allow now operating the device also with magnetic geometries that feature inner-wall limited and diverted ohmic tokamaks. ${ }^{26,28,29}$ Using a toroidal magnetic field on axis $B_{\varphi} \simeq 0.6 \mathrm{~T}$ and a plasma current up to $I_{p} \simeq 150 \mathrm{kA}$, it is possible to perform plasma pulses longer than $1 \mathrm{~s}$ with integrated plasma densities $n_{e} \geq 10^{19} \mathrm{~m}^{-3}$ and core electron temperatures $T_{e} \geq 500 \mathrm{eV}$.

In the following, we consider two circular inboardlimited ohmic L-mode deuterium plasma discharges (\#38373 and \#38413) carried out in the RFX-mod device with a toroidal magnetic field on axis $B_{\varphi}=0.54 \mathrm{~T}$ and plasma currents $I_{p}=150 \mathrm{kA}$ and $I_{p}=100 \mathrm{kA}$. These two plasma currents correspond to $q_{\mathrm{LCFS}}=2$ and $q_{\mathrm{LCFS}}=3$, respectively. The plasma densities and electron temperatures at the LCFS for the two discharges are $n_{e 0}=7.7 \times 10^{17}, 2.0 \times 10^{17} \mathrm{~m}^{-3}$, and $T_{e 0}=16,19 \mathrm{eV}$, respectively, and correspond to the two normalized plasma collisionalities $\nu^{*}=L_{\|} / \lambda^{m f p}=6.9,1.3$, where $L_{\|}=2 \pi q_{L C F S} R$ is the parallel connection length and $\lambda^{m f p}$ is the electron mean free path.

The experimental measurements illustrated in the remaining of this paper are obtained using the U-probe installed in RFX-mod. This probe consists of two boron nitride arms, each of them equipped with 25 electrostatic pins. ${ }^{30,31}$ Fifteen of the pins are used as three five-pin triple probes, ${ }^{32}$ allowing simultaneous measurements of ion saturation, $I_{\text {sat }}$, plasma density, $n$, electron temperature, $T_{e}$, and floating potential, $V_{f}$, while ten others are in the floating mode and used to obtain direct measurements of $V_{f t}$. The time resolution of the measurements is $0.2 \mu \mathrm{s}$. The U-probe is located at a fixed radial position at the outward equatorial midplane, with its arms in the horizontal direction. In order to obtain measurements at different radial locations, the plasma column is shifted towards the inner wall of the device during the discharge, while keeping a constant edge safety factor. We note that the measurements are obtained at approximately $2.8 \mathrm{~cm}$ from the vessel wall. Additionally, we note that for the discharge \#38373 we consider only the experimental measurements taken in between sawtooth crashes. This leads to a reduced number of measurements for the considered time traces (20000 measurements) available for the analysis of the \#38373 discharge (approximately a factor ten less with respect to the \#38413 discharge, for which we have 175000 measurements).

\section{GBS SIMULATIONS OF THE RFX-MOD SOL}

Because of its high collisionality, the tokamak SOL region is generally studied by employing a plasma fluid description, such as the Braginskii fluid model. ${ }^{33}$ Moreover, since the SOL turbulent time scales are much slower than the ion cyclotron time, and the perpendicular (to $\mathbf{B}$ ) scale lengths are longer than the ion Larmor radius, the drift approximation can be applied to simplify the fluid model, thus obtaining a set of drift-reduced Braginskii equations useful to describe the SOL plasma dynamics. ${ }^{34}$ We consider this model also for the present study, although the conditions for the applicability of the fluid model are marginally satisfied for the RFX-mod \#38413 plasma discharge. Neglecting electromagnetic effects as suggested in Ref. 35, since $\beta_{e} R / L_{p} \leq 10^{-3}$ in the RFX-mod SOL ( $\beta_{e}$ is the plasma to magnetic pressure ratio and $L_{p}$ the equilibrium pressure gradient length), assuming an infinite aspect ratio (the influence of finite aspect ratio effects on SOL plasma dynamics is studied in Ref. 36) and cold ions (no ion temperature measurements are available on RFX-mod for these discharges, the impact of ion temperature effects on SOL turbulence is investigated in Ref. 37), and employing the Boussinesq approximation to simplify the vorticity equation (the validity of this assumption in modelling the SOL plasma dynamics is discussed in Refs. 38-40) the drift-reduced Braginskii equations in normalized units are written as

$$
\begin{aligned}
\partial_{t} n= & -R_{0}\{\phi, n\}+2\left[C\left(p_{e}\right)-n C(\phi)\right]-\nabla_{\|}\left(n v_{\| e}\right) \\
& +D_{n} \nabla_{\perp}^{2} n+S_{n}, \\
\partial_{t} \omega= & -R_{0}\{\phi, \omega\}+\frac{2}{n} C\left(p_{e}\right)-v_{\| i} \nabla_{\|} \omega+\frac{1}{n} \nabla_{\| j_{\|}} \\
& +\frac{1}{3 n} C\left(G_{i}\right)+D_{\omega} \nabla_{\perp}^{2} \omega, \\
\partial_{t} v_{\| e}=- & R_{0}\left\{\phi, v_{\| e}\right\}+\frac{m_{i}}{m_{e}}\left[\nabla_{\|} \phi-\frac{1}{n} \nabla_{\|} p_{e}-0.71 \nabla_{\|} T_{e}\right. \\
\left.+\nu j_{\|}-\frac{2}{3 n} \nabla_{\|} G_{e}\right]-v_{\| e} \nabla_{\|} v_{\| e}+D_{v_{\| e}} \nabla_{\perp}^{2} v_{\| e} & \\
& \partial_{t} v_{\| i}=-R_{0}\left\{\phi, v_{\| i}\right\}-v_{\| i} \nabla_{\|} v_{\| i}-\frac{1}{n} \nabla_{\|} p_{e} \\
& -\frac{2}{3 n} \nabla_{\|} G_{i}+D_{v_{\| i}} \nabla_{\perp}^{2} v_{\| i}, \\
\partial_{t} T_{e}=- & R_{0}\left\{\phi, T_{e}\right\}+\frac{4}{3} T_{e}\left[\frac{7}{2} C\left(T_{e}\right)+\frac{T_{e}}{n} C(n)-C(\phi)\right] \\
- & v_{\| e} \nabla_{\|} T_{e}+\nabla_{\|}\left(\chi_{\| e} \nabla_{\|} T_{e}\right) \\
+ & \frac{2}{3} T_{e}\left[0.71 \nabla_{\|} v_{\| i}-1.71 \nabla_{\|} v_{\| e}+0.71\left(\frac{v_{\| i}-v_{\| e}}{n}\right) \nabla_{\|} n\right] \\
+ & D_{T_{e}} \nabla_{\perp}^{2} T_{e}+S_{T_{e}},
\end{aligned}
$$


where $\omega=\nabla_{\perp}^{2} \phi$ is the plasma vorticity, $m_{i} / m_{e}$ is the ion to electron mass ratio, $R_{0}$ is the normalized RFX-mod major radius, $j_{\|}=n\left(v_{\| i}-v_{\| e}\right)$ is the parallel current, $p_{e}=n T_{e}$ is the electron plasma pressure, $\nu$ is the normalized Spitzer resistivity, and $\chi_{\| e}$ is the parallel electron thermal conductivity. The density and electron temperature sources, $S_{n}$ and $S_{T_{e}}$, describe the plasma outflow from the core. The expressions of the two terms representing the ion and electron gyroviscous contributions are given by $G_{i}=-\eta_{0 i}\left[2 \nabla_{\|} v_{\| i}+C(\phi)\right]$ and $G_{e}=-\eta_{0 e}\left[2 \nabla_{\|} v_{\| e}-C\left(p_{e}\right) / n+C(\phi)\right]$, respectively, where $\eta_{0 i}$ and $\eta_{0 e}$ are the normalized gyroviscous coefficients. ${ }^{33}$ The Poisson brackets are defined as $\{\phi, A\}=\mathbf{b} \cdot(\nabla \phi \times \nabla A)$, the curvature operator as $C(A)=B / 2[\nabla \times(\mathbf{b} / B)] \cdot \nabla A$, the parallel gradient as $\nabla_{\|} A=\mathbf{b} \cdot \nabla A$, and the perpendicular Laplacian as $\nabla_{\perp}^{2} A=-\nabla \cdot[\mathbf{b} \times(\mathbf{b} \times \nabla A)]$, with $\mathbf{b}$ the unit vector parallel to $\mathbf{B}$ and $A=n, \omega, \phi, v_{\| i}, v_{\| e}, T_{e}$. Small perpendicular diffusion terms of the form $\mathcal{D}_{A}(A)=D_{A} \nabla_{\perp}^{2} A$ are added for numerical stability reasons. The impact of the perpendicular diffusion terms on typical GBS simulations was investigated in the past, showing that reasonable values (of order unity) of the diffusion coefficients do not modify the results. ${ }^{36}$ Unless specified otherwise, in the present paper, all quantities are normalized to (tilde denotes a physical quantity in $\quad$ SI $\quad$ units): $\quad t=\tilde{t} /\left(\tilde{R} / \tilde{c}_{s 0}\right), n=\tilde{n} / \tilde{n}_{0}, T_{e}=\tilde{T}_{e} / \tilde{T}_{e 0}, \phi$ $=e \tilde{\phi} / \tilde{T}_{e 0}, \quad v_{\| e}=\tilde{v}_{\| e} / \tilde{c}_{s 0}, \quad v_{\| i}=\tilde{v}_{\| i} / \tilde{c}_{s 0}, \quad B=\tilde{B} / \tilde{B}_{0}, \quad R_{0}$ $=\tilde{R} / \tilde{\rho}_{s 0}, \nu=\left(e^{2} \tilde{n}_{0} \tilde{R}\right) /\left(m_{i} \tilde{\sigma}_{\|} \tilde{c}_{s 0}\right)$, where $\tilde{\sigma}_{\|}$is the parallel conductivity, $\tilde{n}_{0}, \tilde{T}_{e 0}$, and $\tilde{B}_{0}$ are the reference density, temperature, and magnetic field, $\tilde{R}$ is the tokamak major radius, and $\tilde{c}_{s 0}$ and $\tilde{\rho}_{s 0}$ are given by $\tilde{c}_{s 0}=\sqrt{\tilde{T}_{e 0} / m_{i}}$ and $\tilde{\rho}_{s 0}=\tilde{c}_{s 0} m_{i} /\left(e \tilde{B}_{0}\right)$. Distances perpendicular to $\mathbf{B}$ are normalized to $\tilde{\rho}_{s 0}$, while parallel distances are normalized to $\tilde{R}$.

Equations (1)-(5) are solved with GBS, a code developed in the last few years to simulate plasma turbulence in the open field region of basic plasma physics experiments and magnetic confinement devices, evolving the full plasma profiles without any separation between equilibrium and perturbation quantities. ${ }^{3,41}$ To develop the GBS code, increasingly complex magnetic configurations were considered. First, the code was developed to describe the plasma dynamics in basic plasma physics experiments, in particular linear devices such as $\mathrm{LAPD}^{42}$ and simple magnetized toroidal devices such as TORPEX. ${ }^{43-45}$ GBS was then extended to the tokamak geometry, and it is now able to model the tokamak SOL region in limited plasmas. ${ }^{12,14,22,35}$ To solve Eqs. (1)-(5), GBS makes use of the toric ( $y$ $=a \theta, x=r, z=\varphi)$ coordinate system, with $\theta$ and $\varphi$ being the poloidal and toroidal angles, and $r$ a flux coordinate. Consequently, considering circular magnetic flux surfaces in the infinite aspect ratio limit and assuming no magnetic shear (a discussion of the impact of these assumptions on DWs and BMs is presented in Refs. 12 and 13), the differential operators can be rewritten as $\{\phi, A\}=\partial_{y} \phi \partial_{x} A$ $-\partial_{x} \phi \partial_{y} A, \quad C(A)=\sin (\theta) \partial_{x} A+\cos (\theta) \partial_{y} A, \quad \nabla_{\|} A=\partial_{z} A$ $+a \partial_{y} A / q$, and $\nabla_{\perp}^{2} A=\partial_{x}^{2} A+\partial_{y}^{2} A$, with $q=q_{L C F S}$. Note that the poloidal angle is defined such that $\theta=0$ and $\theta=2 \pi$ at the outer midplane.
The drift-reduced Braginskii system, Eqs. (1)-(5), is closed by a set of boundary conditions describing the plasma properties at the magnetic pre-sheath entrance. ${ }^{46}$ Within the assumptions used in this section, these boundary conditions are written as

$$
\begin{gathered}
v_{\| i}= \pm c_{s}, \\
v_{\| e}= \pm c_{s} \exp \left(\Lambda-\phi / T_{e}\right), \\
\partial_{y} T_{e}=0, \\
\partial_{y} n=\mp \frac{n}{c_{s}} \partial_{y} v_{\| i}, \\
\omega=-\left(\partial_{y} v_{\| i}\right)^{2} \mp c_{s} \partial_{y}^{2} v_{\| i}, \\
\partial_{y} \phi=\mp c_{s} \partial_{y} v_{\| i},
\end{gathered}
$$

where $\Lambda=\log \sqrt{m_{i} /\left(2 \pi m_{e}\right)} \simeq 3$ for deuterium plasmas. Here, the upper signs apply to the case of the magnetic field directed towards the wall, while the lower ones apply to the opposite case. Equations (1)-(11) are solved using a secondorder finite difference scheme in the spatial dimensions, except for the Poisson's brackets, which are discretized with a second order Arakawa scheme. ${ }^{47}$ Time is advanced using a standard fourth-order Runge-Kutta scheme. For a more detailed description of GBS, we refer to Refs. 3 and 41.

Focusing on a circular plasma with a toroidal limiter located at the high-field side, we perform two nonlinear GBS simulations based on the RFX-mod experimental parameters $R, q_{L C F S}, n_{e 0}$, and $T_{e 0}$. For the two plasma discharges \#38373 and \#38413, these parameters lead to the normalized plasma resistivities $\nu=0.005,0.001$, the normalized major radii $R_{0}=1872,1716$, and the poloidal domain sizes $L_{y}=2700$, 2470. In addition, we consider a reduced ion to electron mass ratio $m_{i} / m_{e}=800$, a reduced normalized parallel electron thermal conductivity $\chi_{\| e}=2$, and the normalized perpendicular diffusion coefficients $D_{A}=5$. The particle and temperature sources, used to mimic the plasma outflow from the core, are assumed poloidally and toroidally constant, with radial dependence $S_{n}(x) \propto S_{T_{e}} \propto \exp \left[-(x-a)^{2} / \sigma^{2}\right]$, being $\sigma=2.5$. We note that the assumption of having a poloidally constant source was investigated in the past showing that, for typical GBS simulations, similar pressure profiles and turbulence properties were found for a poloidally constant source and for a source localized at the low-field side. ${ }^{48}$ The radial domain extends from the inner radius $x_{i}=a-30$ to the outer radius $x_{o}=a+70$ in both simulations. Since a set of first-principle boundary conditions describing the plasma interaction with the outer wall and the interface between the SOL and the core does not exist yet, ad hoc boundary conditions are applied at $x_{i}$ and $x_{o}$, with Neumann's boundary conditions used for $n, v_{\| e}, v_{\| i}$, and $T_{e}$, and Dirichlet's boundary conditions for $\omega$ and $\phi$. To mitigate the impact of these boundary conditions on the simulation results, the two regions extending from $x_{i}$ to $x=a$, and from $x=a+55$ to $x_{o}$ are considered as buffers and are not included in the analysis of the results. This configuration is schematically shown in Fig. 1.

The boundary conditions describing the plasma at the magnetic pre-sheath entrance, Eqs. (6)-(11), are applied at 


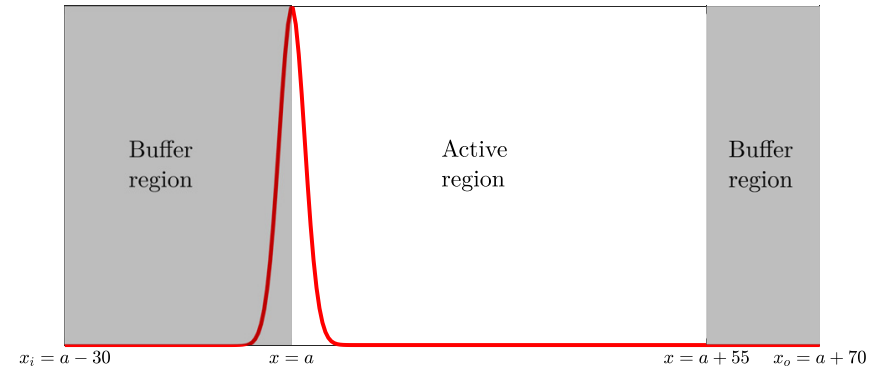

FIG. 1. Schematic of the radial domain considered for the two simulations. The domain extends from $x_{i}=a-30$ to $x_{o}=a+70$, with the regions extending from $x_{i}$ to $x=a$, and from $x=a+55$ to $x_{o}$ acting as buffers. The red thick line represents the particle and temperature sources.

$y=0$ and $y=L_{y}$ for all $x \in\left[x_{i}, x_{o}\right]$. This means that the magnetic field lines in the simulation intercept the limiter also at $x<a$, a region that acts as a buffer and that we exclude from our analysis. We note that edge generated turbulence and the intermittent filaments resulting from it may play an important role in setting the plasma transport near the $\mathrm{LCFS}^{49-51}$ (see Sec. IV for a detailed discussion).

We note that because of the necessary rather large numerical grids $\left(N_{x}, N_{y}, N_{z}\right)=(128,1279,320),(128,1279$, 212 ), with $N_{x}, N_{y}$, and $N_{z}$ the number of points in the radial, poloidal, and toroidal directions, the two simulations discussed herein are extremely expensive in terms of computational resources (approximately one million CPU hours each). The reduced mass ratio and parallel electron thermal conductivity are consequently used to considerably decrease the cost of the simulations. The impact of a reduced mass ratio on the results is investigated in Sec. V B by means of linear simulations. We also note that, while in the experiment the plasma current and the toroidal magnetic field are in the same direction, due to the present GBS limitations, we use a current that is in the opposite direction to the magnetic field in the simulations. We defer the detailed analysis of the impact of the sign of plasma current on SOL turbulence to a future study.

The equilibrium pressure gradient length $L_{p}=-p_{e} / \nabla p_{e}$ is directly evaluated from nonlinear simulations by computing the averaged radial $p_{e}$ profile $p_{e}(y, x)=\left\langle p_{e}(y, x, z, t)\right\rangle_{z, t}$, where $\langle-\rangle_{z, t}$ denotes averaging over $z$ and $t$, and fitting $p_{e}(y$, $x$ ) at fixed $y$ between $x=a$ and $x=a+55$ assuming $p_{e}(y, x) \propto \exp \left[-(x-a) / L_{p}(y)\right]$. For the two plasma discharges \#38373 and \#38413, we find at the outer midplane
$L_{p}(0)=31$ and $L_{p}(0)=37$, respectively. Moreover, it is possible to estimate the poloidal wave number of the mode that drives most of the turbulent transport by computing in the nonlinear simulations the $p_{e}$ and $\phi$ fluctuations, $\delta p_{e}$ and $\delta \phi$ (see Fig. 2). For the two plasma discharges considered herein, we find $k_{y} \approx 0.1-0.2$.

\section{VALIDATION OF THE GBS SIMULATIONS AGAINST EXPERIMENTAL MEASUREMENTS}

In order to assess the reliability of the drift-reduced Braginskii model and of the GBS simulations, we compare the nonlinear numerical results with the RFX-mod experimental measurements. We remark that the plasma dynamics inside the LCFS is neglected in the simulations considered herein. Therefore, we expect a better agreement between the simulation results and experimental measurements in the far SOL than in the near SOL. Indeed, the simulations display a shearing rate in the proximity of the LCFS that is larger than the linear growth rate. However, we refrain ourselves from carrying out an analysis of the expected steepening of the pressure profile in the near SOL due to the velocity shear, ${ }^{52}$ since we expect the dynamics across the LCFS not to be well captured by the simulations. Note that the results illustrated in the present section are in SI units.

First, in Fig. 3 we present the experimental and numerical radial equilibrium profiles of $n, T_{e}, j_{s a t}$, and $V_{f l}$ for the two RFX-mod discharges discussed above (we evaluate $j_{s a t}=e n c_{s} / 2$ and $V_{f l}=\phi-\Lambda T_{e} / e$ at the outer midplane in the simulations). According to the results presented in Ref. 53, we assume a $20 \%$ relative discretization error affecting the simulation equilibrium profiles and we neglect other sources of uncertainties. We observe that the values of $n, T_{e}$, and $j_{\text {sat }}$ obtained from the nonlinear simulations of both discharges are consistent with the experimental results within the estimated uncertainties at the measurements points in the far SOL. Moreover, we note that, since we are enforcing $n=n_{0}$ and $T_{e}=T_{e 0}$ at the LCFS in the simulations, this also corresponds to comparing the averaged gradient of $n, T_{e}$, and $j_{\text {sat }}$ in the SOL. Concerning $V_{f}$, the simulation results do not agree with the experimental measurements, in particular in the proximity of the LCFS. As a matter of fact, while the experimental measurements are in agreement with observations in other devices (see, e.g., Ref. 54), with a strong drop of $V_{f}$ in the proximity of the LCFS extending for a few
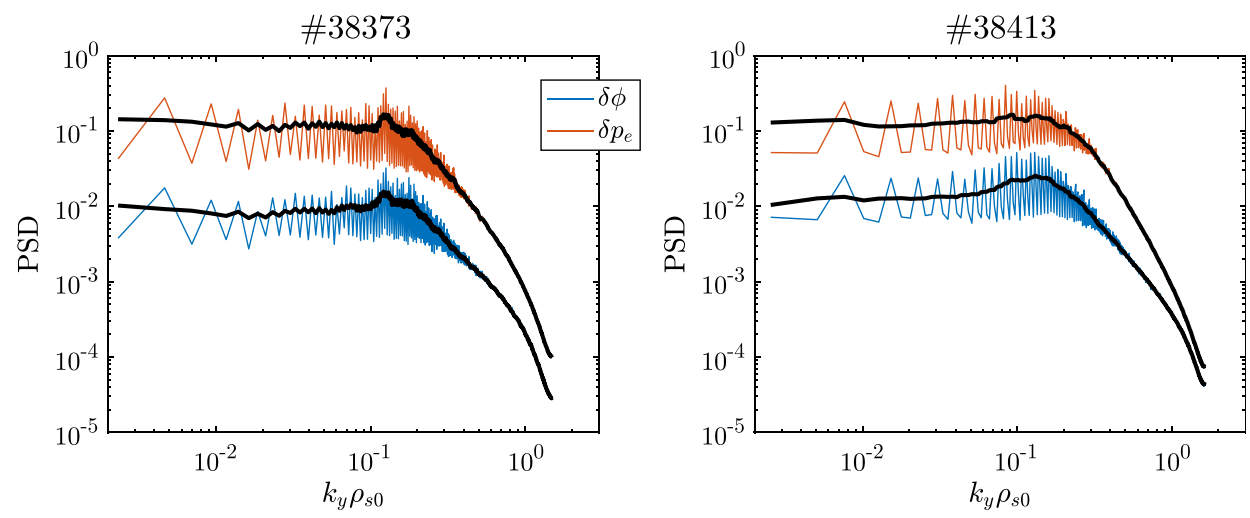

FIG. 2. PSD of $\delta \phi$ (blue lines) and $\delta n$ (red lines) for the two simulations of the RFX-mod plasma discharges \#38373 (left) and \#38413 (right) at the outer midplane, at approximately $2 \mathrm{~cm}$ from the LCFS. The black lines denote a smoothing of the PSD. 

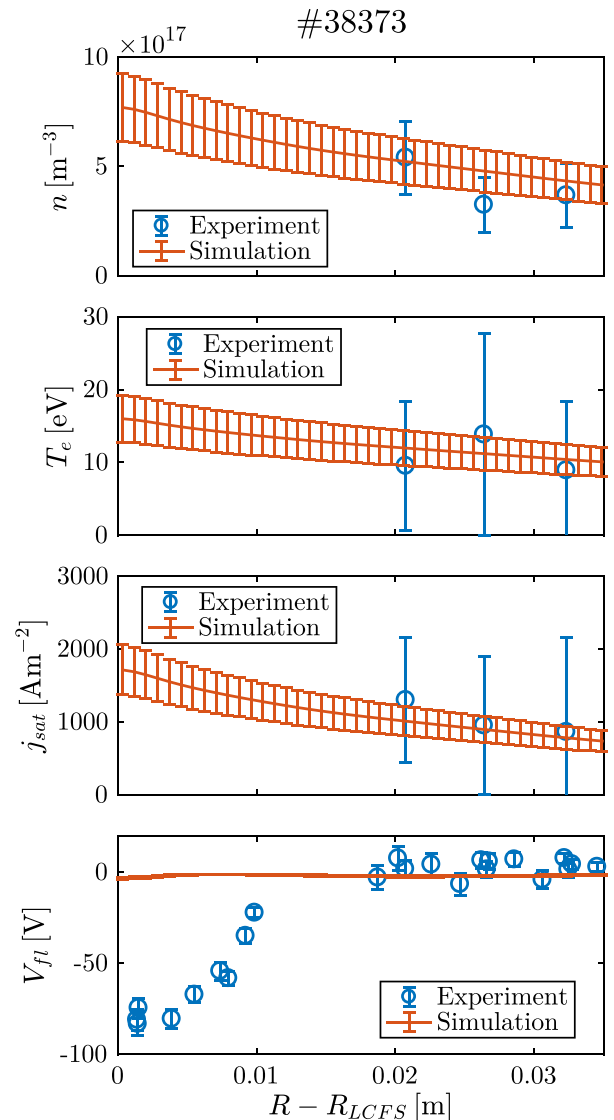
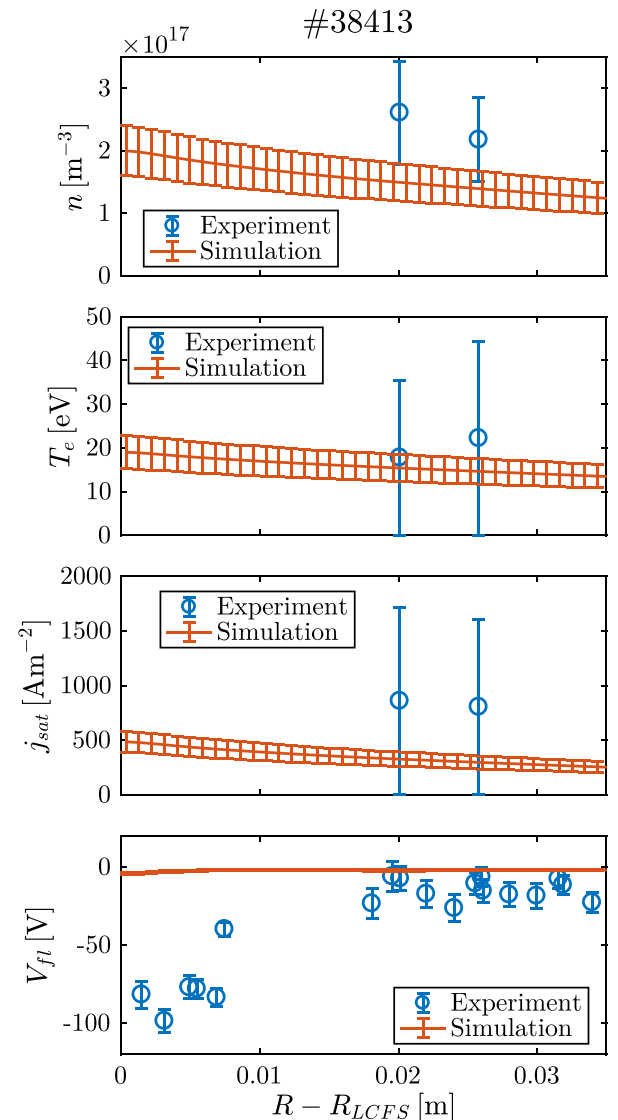

FIG. 3. Experimental (blue circles) and simulation (red lines) radial equilibrium profiles of plasma density (first row), electron temperature (second row), ion saturation current density (third row), and floating potential (fourth row), for the two RFX-mod plasma discharges \#38373 (left column) and \#38413 (right column). millimeters in the SOL, the simulation profiles are flatter, with an absolute value of $V_{f l}$ closer to zero. This discrepancy is probably related to simulating only the open field line region of RFX-mod, since the plasma dynamics close to the LCFS plays an important role in setting $V_{f}$ in the near SOL, ${ }^{52}$ and to neglecting ion temperature effects.

In Fig. 4, we compare the experimental root-meansquare (RMS) values of $j_{\text {sat }}$ fluctuations, $\delta j_{\text {sat }}^{R M S}$, normalized to the equilibrium $j_{\text {sat }}$, with the simulation results. We observe that the simulations underestimate the $j_{\text {sat }}$ fluctuations approximately by a factor of two in both considered discharges. In Fig. 4, we also display the skewness and the kurtosis of the experimental and numerical $j_{\text {sat }}$ time traces. For these quantities, the simulation results show a better agreement with the experimental measurements than for $\delta j_{\text {sat }}^{R M S}$. In particular, the simulation results display a $j_{\text {sat }}$
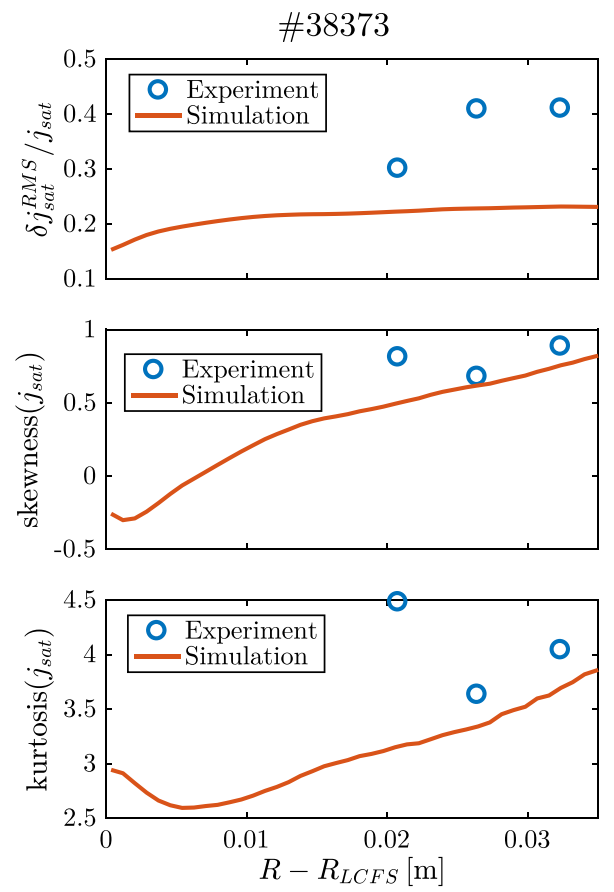
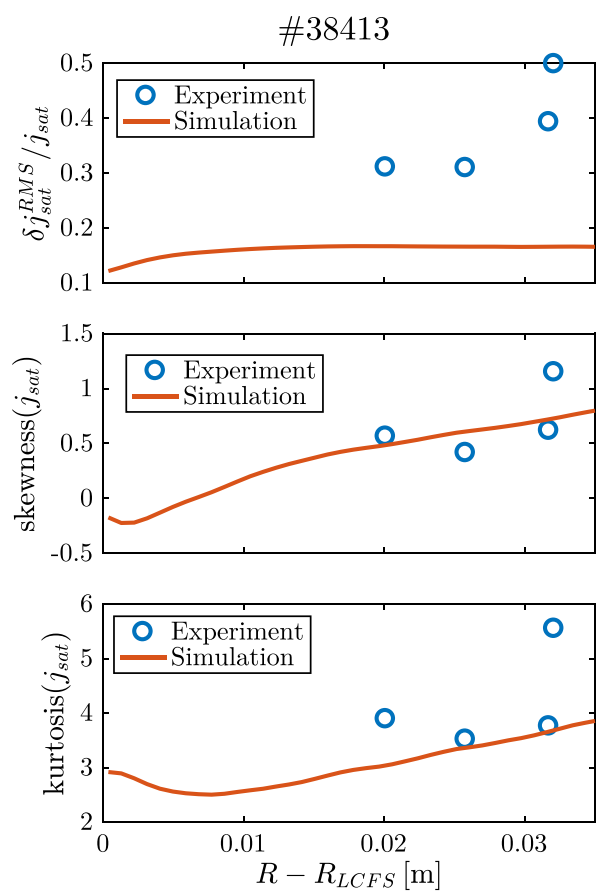

FIG. 4. Experimental (blue circles) and simulation (red lines) radial profiles of $\delta j_{\text {sat }}^{R M S} / j_{\text {sat }}$ (first row), $j_{\text {sat }}$ skewness (second row), and $j_{\text {sat }}$ kurtosis (third row), for the two RFX-mod plasma discharges \#38373 (left column) and \#38413 (right column). 

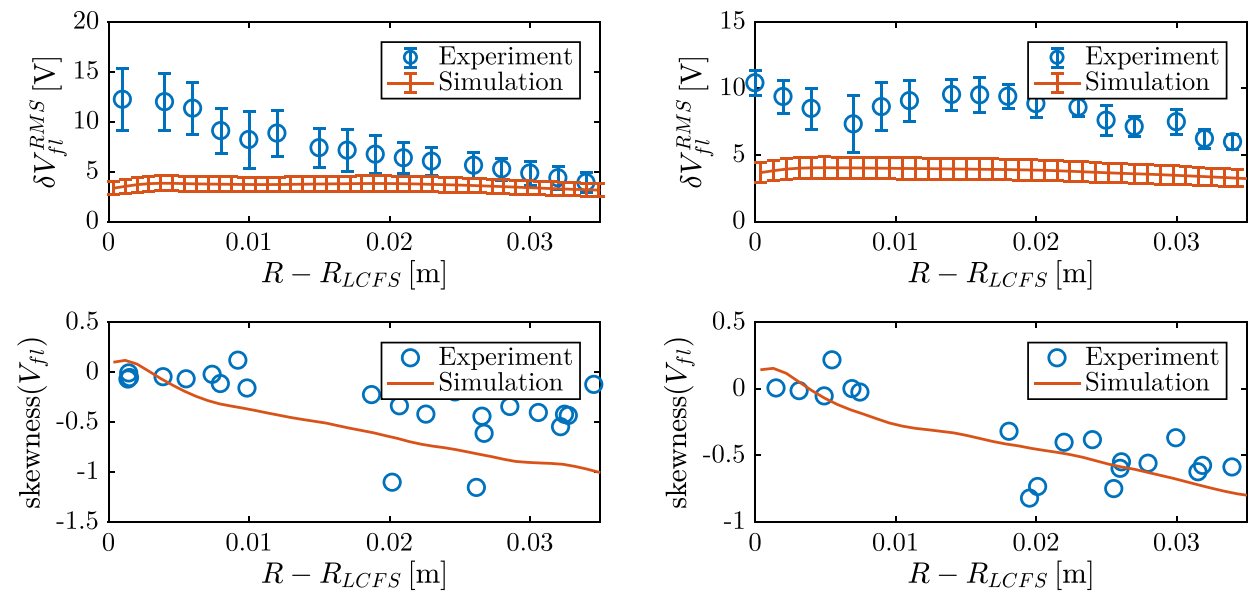

FIG. 5. Experimental (blue circles) and simulation (red lines) radial profiles of $\delta V_{f l}^{R M S}$ (first row), $V_{f l}$ skewness (second row), and $V_{f}$ kurtosis (third row), for the two RFX-mod plasma discharges \#38373 (left column) and \#38413 (right column).
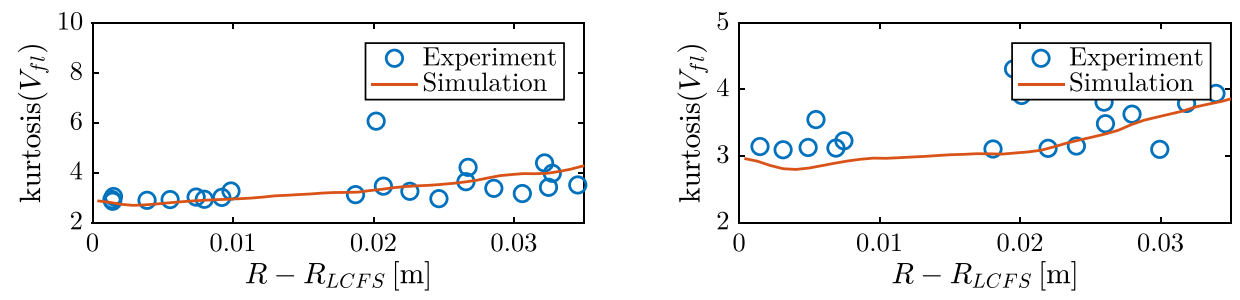

skewness close to zero in the proximity of the LCFS and monotonically increasing in the SOL, in agreement with previous experimental SOL investigations. ${ }^{55-57}$

In Fig. 5 we present the radial profiles of the $V_{f l}$ fluctuation RMS values, $\delta V_{f l}^{R M S}$, and of the $V_{f l}$ skewness and kurtosis. Concerning the RMS values, we observe an almost radial constant level of fluctuations in both simulations. On the other hand, the experimental measurements show an almost monotonically decreasing profile in the SOL and a better agreement with the simulation results in the far SOL. We note that, in general, the numerical results display a better quantitative agreement with the RFX-mod experimental measurements for the \#38373 discharge, and that the agreement worsens considering the discharge with lower plasma collisionality. The $V_{f}$ skewness monotonically decreases in the SOL both for the simulations and for the RFX-mod experimental measurements, with good quantitative agreement between the two quantities. Finally, concerning the $V_{f}$ kurtosis, we observe good qualitative agreement for both discharges, with an almost constant value close to three, except for $R-R_{L C F S}>2.5 \mathrm{~cm}$, where the kurtosis is larger. We note that a comparison of the $V_{f l}$ moments between simulations and experimental measurements is also discussed in Ref. 58 considering TORPEX plasma discharges. Considerably larger discrepancies between numerical results and experimental measurements were found in that case, probably due to the presence of fast electrons, resulting from the source operating at the electron cyclotron resonance.

Our observations on the agreement of $j_{\text {sat }}$ and $V_{f l}$ skewness and kurtosis are confirmed by comparing the numerical and experimental probability density functions (PDF) corresponding to $j_{\text {sat }}$ and $V_{f l}$ fluctuations, $\delta j_{\text {sat }}$ and $\delta V_{f}$, normalized to their standard deviation in the far SOL, at approximately $2 \mathrm{~cm}$ from the LCFS, as shown in Fig. 6. We observe that the simulation results are in good agreement with the experimental measurements for both physical quantities and for both discharges. The $\delta j_{\text {sat }}$ PDF displays a positive skewness, while the $\delta V_{f l}$ PDF is negatively skewed. We note that small differences are observed between experimental measurements and simulation results in the PDF tails, particularly for the plasma discharge \#38373. This could be due to intermittent events, originated inside the LCFS that are not taken into account in the simulations. However, these differences are too small to explain the different level of $j_{\text {sat }}$ fluctuations, and they allow us to conclude that the different levels of fluctuations between simulations and experimental measurements are not related to coherent intermittent events, which would strongly affect the PDF tails.

A comparison of the experimental and simulation $j_{\text {sat }}$ and $V_{f l}$ time traces at $2 \mathrm{~cm}$ from the LCFS, normalized to

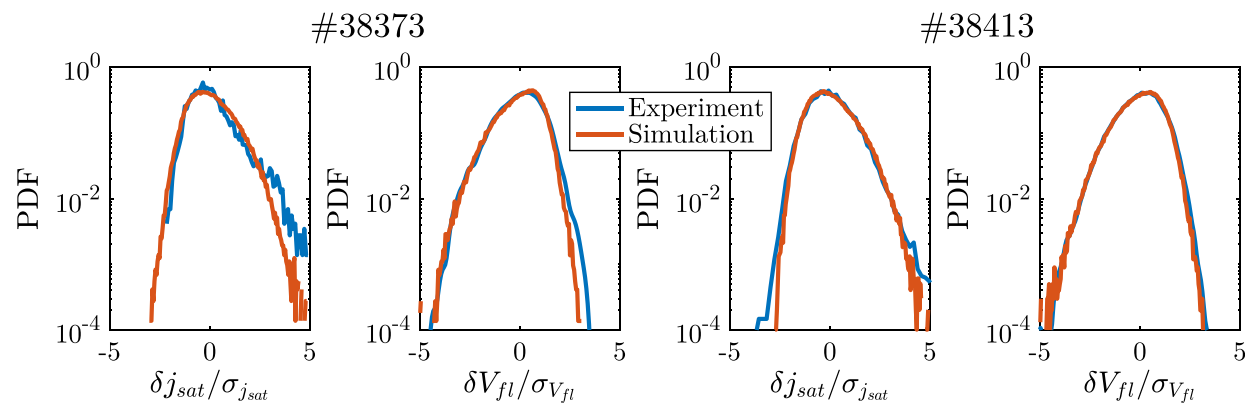

FIG. 6. Experimental (blue lines) and simulation (red lines) PDF of $\delta j_{s a t}$ (first and third columns) and $\delta V_{f l}$ (second and fourth columns) normalized to their standard deviation. The results are evaluated approximately at $2 \mathrm{~cm}$ from the LCFS and are displayed for the two RFX-mod plasma discharges \#38373 (first and second columns) and \#38413 (third and fourth columns). 

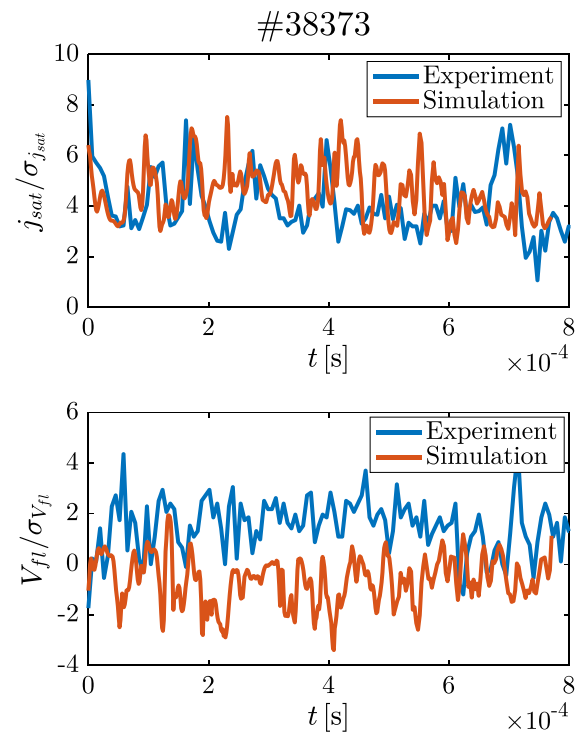

their standard deviation, is presented in Fig. 7. We observe that the duration and the interval between burst events are comparable in the two cases.

To gain a deeper insight into the nature of the instability driving most of the SOL turbulent transport, in Fig. 8 we compare the experimental joint probabilities between $\delta j_{\text {sat }}$ and $\delta V_{f l}$ at approximately $2 \mathrm{~cm}$ from the LCFS, normalized to their standard deviation, with the simulation results. A good qualitative agreement between experimental measurements and simulation results is found, with $\delta j_{s a t} / \sigma_{j_{s a t}}$ and $\delta V_{f l} / \sigma_{V_{f l}}$ showing moderate anticorrelation.

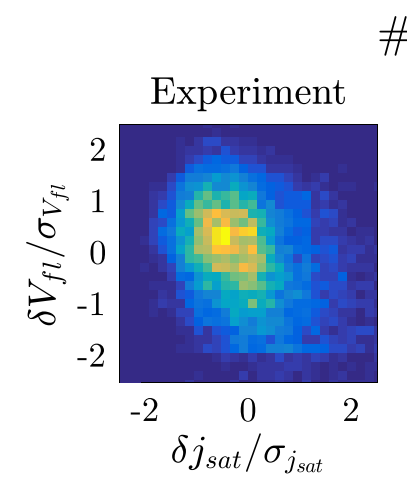

\#38373

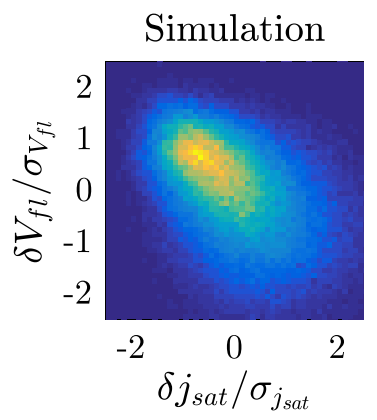

\#38413
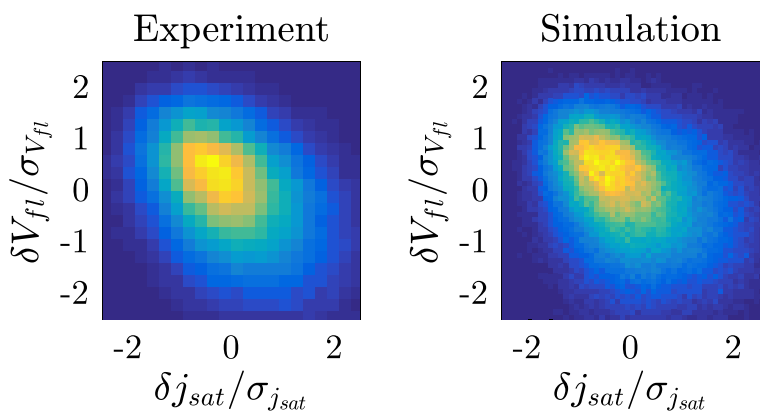

FIG. 8. Experimental (first column) and simulation (second column) joint probabilities of $\delta j_{s a t}$ and $\delta V_{f t}$ normalized to their standard deviation. The results are evaluated approximately at $2 \mathrm{~cm}$ from the LCFS and are displayed for the two RFX-mod plasma discharges \#38373 (first row) and \#38413 (second row).
FIG. 7. Experimental (blue lines) and simulation (red lines) time traces of $j_{\text {sat }}$ (first line) and $V_{f l}$ (second line) normalized to their standard deviation. The results are evaluated approximately at $2 \mathrm{~cm}$ from the LCFS and are displayed for the two RFX-mod plasma discharges \#38373 (first column) and \#38413 (second column).
For the analysis of the equilibrium profiles and fluctuation properties, it emerges that the major difference between experimental measurements and simulations lies in the level of $j_{\text {sat }}$ fluctuations. We explore the reason of this discrepancy in Fig. 9, where we display the numerical and experimental PSD of $j_{\text {sat }}$ and $V_{f l}$ at approximately $2 \mathrm{~cm}$ from the LCFS. We observe that for both discharges and for both quantities the PSD monotonically decreases for $f \gtrsim 10 \mathrm{kHz}$, in agreement with previous observations. ${ }^{20}$ However, the simulation PSD is smaller than the experimental one, particularly for the \#38413 discharge, whose plasma collisionality is smaller, consistently with the $\delta j_{\text {sat }}^{R M S}$ and $\delta V_{f l}^{R M S}$ observations. In addition, while in the simulations the $j_{\text {sat }}$ and $V_{f l}$ spectral-slopes, $\alpha_{j s a t}$ and $\alpha_{V f l}$, are very similar, with $\alpha_{j s a t} \simeq \alpha_{V f l} \simeq-2.4 \mathrm{~s}$ for $10 \mathrm{kHz} \lesssim f \lesssim 100 \mathrm{kHz}$ and $\alpha_{j s a t} \simeq \alpha_{V f l} \simeq-4.1 \mathrm{~s}$ for $f \gtrsim 100 \mathrm{kHz}$, in the experiment we have $\alpha_{j s a t} \simeq \alpha_{V f l} \simeq-1.0 \mathrm{~s}$ for $10 \mathrm{kHz} \leqq f \leqq 100 \mathrm{kHz}$ and $\alpha_{j s a t} \simeq-2.5 \mathrm{~s}$ and $\alpha_{V f l} \simeq=-2.9 \mathrm{~s}$ for $f \gtrsim 100 \mathrm{kHz}$. We note that $\alpha_{j s a t} \neq \alpha_{V f l}$ is observed also in other experimental devices (see, e.g., Ref. 59). In Fig. 9 we also display the phase shift and the coherence between $j_{\text {sat }}$ and $V_{f l}$ fluctuations. First, we note that the experimental measurements are noisier for the \#38373 discharge because of the presence of sawtooth instabilities and of the resulting lower temporal statistics used for the analysis. We also observe that the phase shift between $\delta V_{f l}$ and $\delta j_{\text {sat }}, \mathcal{P}\left(\delta V_{f l}, \delta j_{\text {sat }}\right)$, resulting from the nonlinear simulations is in better agreement with experimental measurements for $f \gtrsim 20 \mathrm{kHz}$ than it is at low frequencies. As a matter of fact, in the simulations we find $\mathcal{P}\left(\delta V_{f l}, \delta j_{\text {sat }}\right)<0$ at all frequencies, while in the experiment $\mathcal{P}\left(\delta V_{f l}, \delta j_{\text {sat }}\right)>0$ for $f \leqq 20 \mathrm{kHz}$. This discrepancy at low frequencies seems related to incoherent experimental fluctuations, as shown in the last row of Fig. 9. In fact, the simulation results display a quite strong coherence between $\delta j_{\text {sat }}$ and $\delta V_{f}$ at all frequencies, while the experimental measurements show a lower coherence, particularly at low frequencies. Overall, the results presented in Fig. 9 indicate a better agreement between simulations and experimental measurements in the frequency range $10 \mathrm{kHz} \lesssim f \lesssim 100 \mathrm{kHz}$, where the coherence between $\delta j_{\text {sat }}$ and $\delta V_{f l}$ is higher, while the agreement worsen at low and 

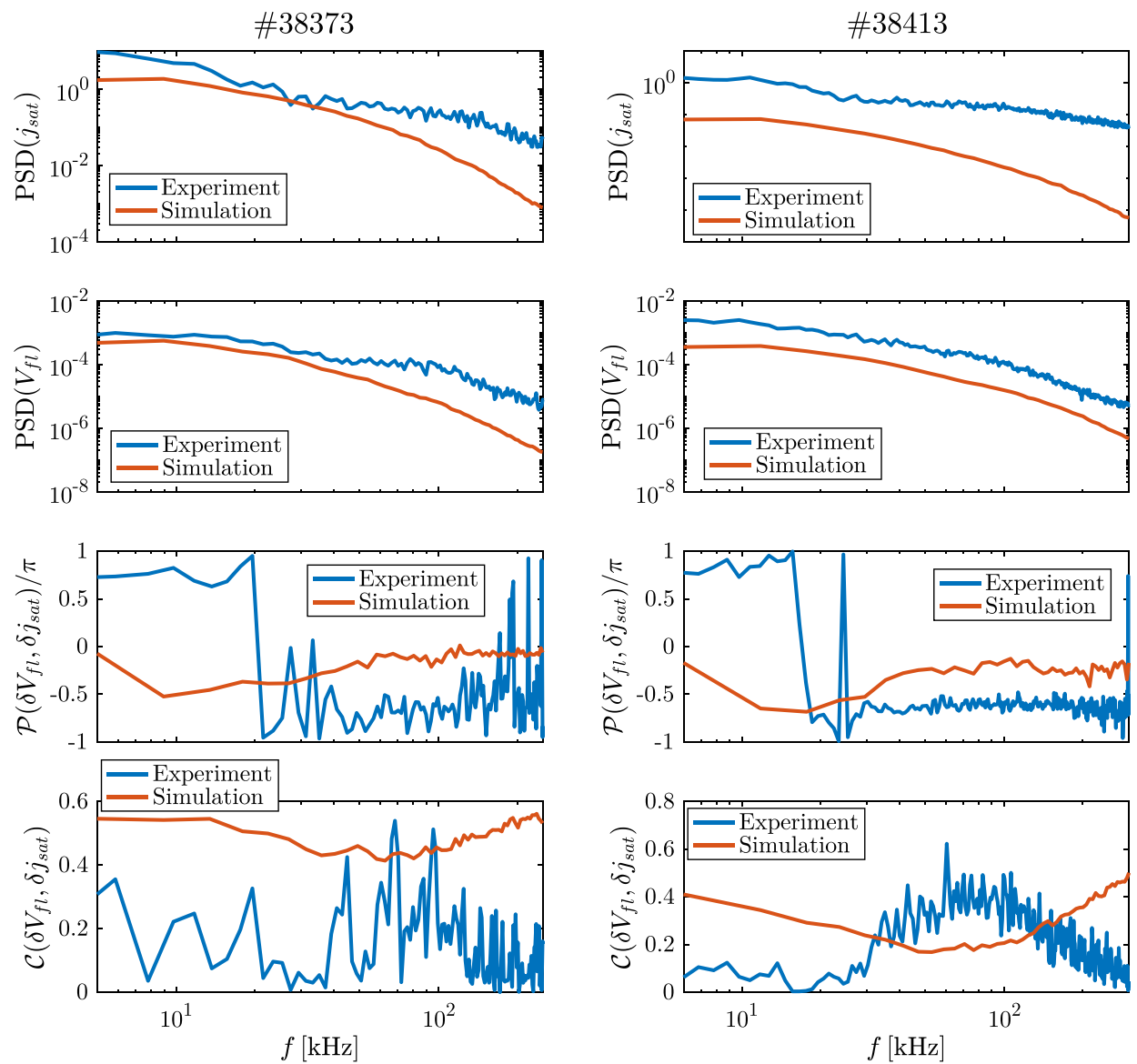

FIG. 9. Experimental (blue lines) and simulation (red lines) $j_{\text {sat }}$ (first row) and $V_{f l}$ (second row) PSD, and phase shift (third row) and coherence (fourth row) between $\delta j_{\text {sat }}$ and $\delta V_{f f}$, for the two RFX-mod plasma discharges \#38373 (left column) and \#38413 (right column), at approximately $2 \mathrm{~cm}$ from the LCFS. high frequencies, with the RFX-mod measurements dominated by incoherent fluctuations.

To further investigate the discrepancies observed between simulations and experimental measurements, in Fig. 10 we display the $S\left(k_{\theta}, f\right)$ spectra obtained from the $V_{f}$ time traces at $R-R_{L C F S} \simeq 2 \mathrm{~cm}$ and related to the two plasma discharges \#38373 and \#38413. We note that, while the spectra obtained from the $V_{f l}$ experimental measurements are evaluated according to the two-point correlation technique described in Ref. 60, the simulation results are obtained computing the Fourier transform of the $V_{f t}$ time signals along $t$ and $y$. In Fig. 10 we observe that the modes are mainly rotating in the ion diamagnetic direction, both in the experiment and in the simulations. However, while in the experiment the dominant modes have $k_{\theta} \rho_{s 0} \leqq 0.03$, for the simulations $0.1 \leq k_{\theta} \rho_{s 0} \leq 0.2$, as also shown in Sec. III. We note that, assuming a linear relation between $k$ and $f$, in the simulations we obtain $k_{\theta} \gtrsim 1 / \rho_{s 0}$ for $f \gtrsim 100 \mathrm{kHz}$. Since the drift approximation is not justified for $k_{\theta} \rho_{s 0} \gtrsim 1$, and $k_{\theta} \rho_{s 0}=1$ corresponds approximately to the maximum wave number resolved by the grids used for the present simulations, we infer that the increasing discrepancy observed for $f \gtrsim 100 \mathrm{kHz}$ in the PSD may be related to the limits of the drift-reduced Braginskii model and to the simulation finite grid resolution.

In summary, the GBS model is able to qualitatively reproduce most of the RFX-mod experimental measurements, with the noteworthy exception of $\delta j_{\text {sat }}$ and, in general, a better agreement for the RFX-mod plasma discharge
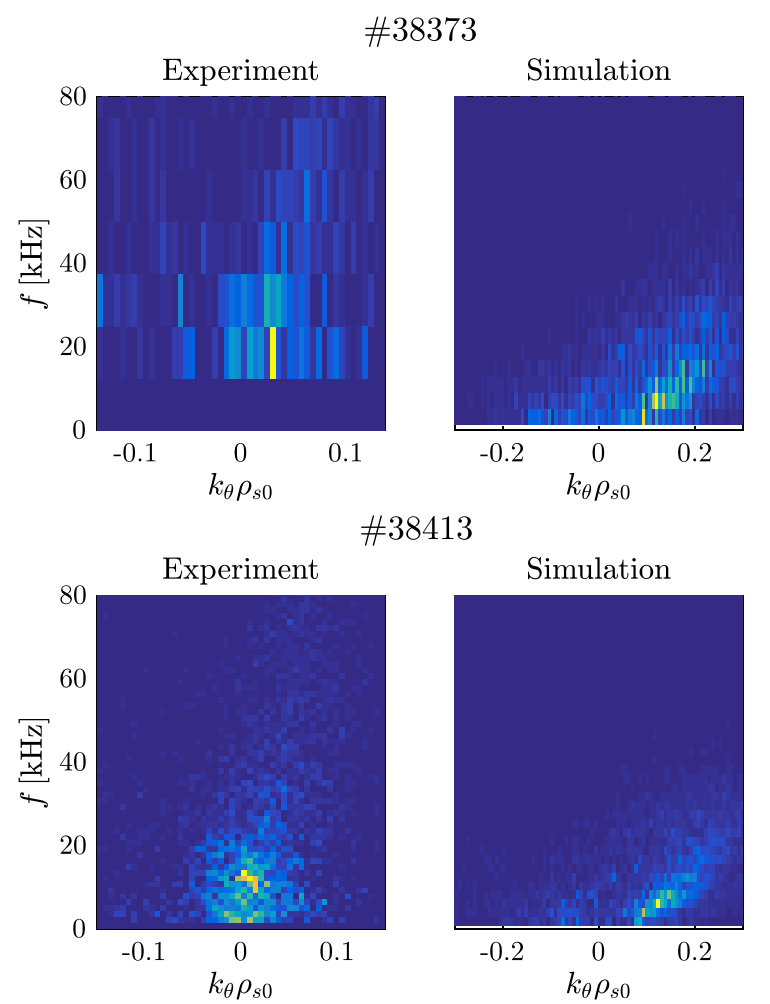

FIG. 10. Experimental (first column) and simulation (second column) $S\left(k_{\theta}\right.$, $f$ ) spectra obtained from $V_{f}$ time-traces for the two RFX-mod plasma discharges \#38373 (first row) and \#38413 (second row). 
\#38373, whose plasma collisionality is higher than in the \#38413 discharge. Since the $\delta j_{\text {sat }}$ and $\delta V_{f t}$ phase shift and joint probability agree between the simulation results and experimental measurements at the frequencies where the fluctuations are more coherent, we infer that the nature of the SOL turbulent transport in the simulations and in the experiment should be the same, with coherent structures having similar statistical properties.

The differences observed in the $V_{f}$ radial profile and in the level of $j_{\text {sat }}$ fluctuations may be, at least in part, related to simulating only the tokamak SOL region, neglecting the plasma dynamics inside the LCFS. As a matter of fact, we note that previous tests performed considering GBS simulations of ISSTOK ${ }^{61}$ indicate an increase of $\delta j_{\text {sat }}^{R M S} / j_{\text {sat }}$ up to $30 \%$ when the plasma dynamics inside the LCFS is included in the simulations. In addition, sensitivity tests pointed out that $\delta j_{\text {sat }}^{R M S}$ depends on the plasma resistivity, with $\delta j_{\text {sat }}^{R M S}$ increasing by approximately $15 \%$ when increasing $\nu$ by a factor ten.

\section{IDENTIFICATION OF THE INSTABILITY DRIVING THE SOL TRANSPORT}

Previous investigations of the SOL plasma dynamics indicate DWs and BMs as the main instabilities driving SOL turbulent transport. ${ }^{12,35,62} \mathrm{BMs}$ are interchange-like modes, driven unstable, in the presence of plasma resistivity and electron inertia, by the magnetic curvature and pressure gradients pointing in the same direction. ${ }^{11,63-66}$ DWs are due to an $\mathbf{E} \times \mathbf{B}$ convection of the plasma pressure when electron adiabaticity is broken by resistivity or finite electron mass, leading, respectively, to resistive DWs (RDW) and InDWs. ${ }^{67-71}$

Previous studies show that $q_{L C F S}$ and $\nu$ strongly affect the SOL turbulent regime. In particular, it is demonstrated that there exists a threshold value of $\nu$ below which a transition from resistive BMs (RBM) to InDWs is observed, and this threshold value increases with the decrease of the edge safety factor. ${ }^{12}$ While in typical tokamak conditions, the SOL is expected to be in the RBM regime or marginally in the DW regime, for the parameters considered herein turbulence is expected to be clearly in the InDW regime. ${ }^{12}$

The comparison of the nonlinear simulations against RFX-mod experimental measurements in Sec. IV shows good agreement for most of the considered quantities. This comparison allows us to infer that the SOL turbulent transport is mostly driven by the same instability in the experiment and in the simulations. Consequently, in the following of this section, we investigate the nature of the instability that drives most of the SOL turbulent transport in the RFXmod plasma discharges \#38373 and \#38413 by using nonlinear simulations and, also, the linear theory.

\section{A. Nonlinear simulations}

In order to identify the instability that drives most of the RFX-mod SOL turbulent transport, we proceed as follows. Considering the plasma discharge \#38373, we perform three nonlinear simulations solving (i) the "full" GBS model, Eqs. (1)-(5), (ii) the "BM" model, considering Eqs. (1)-(5), where we neglect the diamagnetic term in the Ohm's equation, i.e., we simplify Eq. (3) as

$$
\begin{aligned}
\partial_{t} v_{\| e}= & -\frac{R}{\rho_{s 0}}\left\{\phi, v_{\| e}\right\}+\frac{m_{i}}{m_{e}}\left[\nabla_{\|} \phi+\nu j_{\|}-\frac{2}{3 n} \nabla_{\|} G_{e}\right] \\
& -v_{\| e} \nabla_{\|} v_{\| e}+D_{v_{\| e}} \nabla_{\perp}^{2} v_{\| e}
\end{aligned}
$$

and (iii) the "DW" model, where we neglect the pressure curvature term in the vorticity equation of the "full" GBS model, which corresponds to rewriting Eq. (2) as

$$
\begin{aligned}
\partial_{t} \omega= & -\frac{R}{\rho_{s 0}}\{\phi, \omega\}-v_{\| i} \nabla_{\|} \omega+\frac{1}{n} \nabla_{\| j_{\|}}+\frac{1}{3 n} C\left(G_{i}\right) \\
& +D_{\omega} \nabla_{\perp}^{2} \omega .
\end{aligned}
$$

For each simulation, we then compute $L_{p}(y)$ following the procedure described in Sec. III. The values of $L_{p}(y)$ thus obtained are shown in Fig. 11 for the three models. We observe that the "full" and the "DW" models lead to quite similar $L_{p}$ for $y>0$, while $L_{p}$ is larger for $y<0$ in the "DW" simulations, particularly in the proximity of the limiter. This is probably due to the stabilizing effect of magnetic curvature on SOL turbulence at the tokamak high-field side. On the other hand, the value of $L_{p}$ for the "BM" model is smaller than in the original simulation for all $y$. This suggests that DWs are driving most of the SOL turbulent transport, and therefore are responsible for the flattening of the pressure profile, in agreement with the expectations in Ref. 12.

We note that, because of the extremely high computational cost of the simulations, we did not perform simulations with the "BM" and "DW" models for the \#38413 discharge. However, according to Ref. 12, the same nature of the SOL turbulence is expected for the two discharges considered herein.

\section{B. Linear instabilities}

As a confirmation of the nature of the turbulent transport identified by using the nonlinear simulations, we consider the linear properties of the instability dominating the SOL plasma dynamics. This approach allows us also to disentangle more easily the role of resistivity and electron inertia and

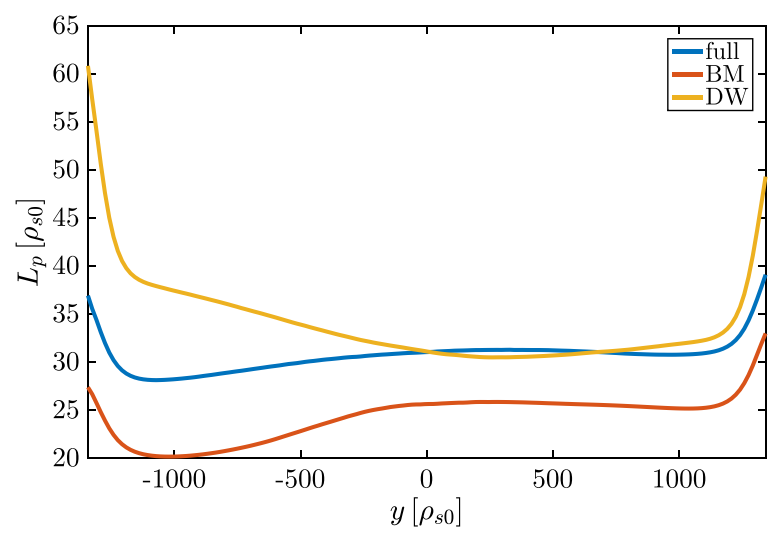

FIG. 11. Profiles of $L_{p}$ as a function of $y$ based on the RFX-mod discharge \#38373, solving the "full" GBS model, Eqs. (1)-(5) (blue line), the "BM" model (red line), and the "DW" model (yellow line). 
to study the realistic ion to electron mass ratio not accessible by the nonlinear simulations.

The linear model we consider for this study is detailed in Sec. III of Ref. 72. Considering circular magnetic geometries in the infinite aspect ratio, the resulting system of equations writes

$$
\begin{gathered}
\gamma \delta n=-i k_{Y} \frac{R_{0}}{L_{n}} \delta \phi-2 i k_{Y} \cos (\theta)\left(\delta p_{e}-\delta \phi\right)+\partial_{Z}\left(\delta j_{\|}-\delta v_{\| i}\right) \\
\gamma \delta \omega=-2 i k_{Y} \cos (\theta) \delta p_{e}+\partial_{Z} \delta j_{\|}, \\
\frac{m_{e}}{m_{i}} \gamma \delta v_{\| e}=\partial_{Z}\left(\delta \phi-\delta p_{e}-0.71 \delta T_{e}\right)+\nu \delta j_{\|} \\
\gamma \delta T_{e}=-i k_{Y} \eta \frac{R_{0}}{L_{n}} \delta \phi-i k_{Y} \frac{4 \cos (\theta)}{3}\left(\delta p_{e}+\frac{5}{2} \delta T_{e}-\delta \phi\right) \\
+\frac{2}{3} \partial_{Z}\left(1.71 \delta j_{\|}-\delta v_{\| i}\right)
\end{gathered}
$$

where $k_{Y}$ is the poloidal wave number, $\gamma$ is the linear growth rate, $Z$ is a parallel (to $\mathbf{B}$ ) coordinate, $\eta=L_{n} / L_{T_{e}}$, with $L_{n}$ and $L_{T_{e}}$ being the characteristic lengths associated with $n$ and $T_{e} \quad$ at the LCFS, $\delta p_{e}=\delta n+\delta T_{e}, \quad \delta j_{\|}=\delta v_{\| i}-\delta v_{\| e}$, $\delta \omega=-k_{Y}^{2} \delta \phi$. Equations (14)-(18) determine the linear growth rate of the SOL plasma instabilities. To solve Eqs. (14)-(18), a numerical code was developed, which evaluates $\gamma$ as a function of the parameters $R_{0} / L_{n}, \eta, \nu, q$, and $k_{Y}$, as detailed in Ref. 72.

As discussed in Sec. V A, it is possible to remove the BM instability from the system, Eqs. (14)-(18), by zeroing out the curvature term in the vorticity equation, i.e., neglecting the first term of the right-hand side of Eq. (15). The solution of the resulting reduced model is denoted in the following as $\gamma_{D W}$. On the other hand, similar to Eq. (12), DWs are removed from the model by neglecting the diamagnetic term in the Ohm's equation, i.e., zeroing out the $\delta p_{e}$ and $\delta T_{e}$ terms of Eq. (16). The solution of this reduced model is denoted in the following as $\gamma_{B M}$.

Considering $m_{e} / m_{i}=800$ and the parameters $\nu, R_{0}$, and $q$ provided by the experimental measurements of the plasma equilibrium, setting $\eta \simeq 0.7$ according to typical simulation results (see, e.g., Ref. 43, and also in agreement with the nonlinear results obtained with the two GBS simulations of RFX-mod), and imposing $L_{p}$ and $k_{Y}$ as evaluated in Sec. III from the nonlinear simulations, we solve Eqs. (14)-(18) for $\gamma, \gamma_{D W}$, and $\gamma_{B M}$. For the two discharges \#38373 and \#38413, we obtain $\gamma=5.1,4.4, \gamma_{D W}=5.2,4.5$, and $\gamma_{B M}=0.3,0.1$, respectively. While the values of $\gamma_{D W}$ are similar to the growth rates obtained by solving the original Eqs. (14)-(18), removing the DWs from the system leads to a growth rate close to zero. This means that the DW is the instability that drives most of the SOL turbulent transport in the two plasma discharges considered herein, in agreement with the nonlinear results and theoretical expectations.

In order to disentangle the impact of resistivity and electron inertia on DWs, we simplify Eqs. (14)-(18) as follows. We first neglect the curvature terms to avoid coupling with
BMs, together with the compressibility terms in the continuity and temperature equations. Then, assuming $\gamma \gg k_{Z}$, we remove the sound wave coupling from the model. The resulting system of equations is written as

$$
\begin{gathered}
\gamma \delta n=-i k_{Y} \frac{R_{0}}{L_{n}} \delta \phi-\partial_{Z} \delta v_{\| e} \\
\gamma \delta \omega=-\partial_{Z} \delta v_{\| e} \\
\frac{m_{e}}{m_{i}} \gamma \delta v_{\| e}=\partial_{Z}\left(\delta \phi-\delta p_{e}-0.71 \delta T_{e}\right)-\nu \delta v_{\| e} \\
\gamma \delta T_{e}=-i k_{Y} \eta \frac{R_{0}}{L_{n}} \delta \phi-1.71 \frac{2}{3} \partial_{Z} \delta v_{\| e} .
\end{gathered}
$$

Equations (19)-(22) constitute the minimal model necessary to describe the linear dynamics of RDWs and InDWs. RDWs and InDWs are removed from the model, Eqs. (19)-(22), by setting $\nu=0$ and $m_{e} / m_{i}=0$, respectively. Solving Eqs. (19)-(22) for the two plasma discharges \#38373 and \#38413 with the linear code discussed above, we obtain respectively $\gamma=6.1,4.7$ for $\nu=0$ and $\gamma=3.1,1.9$ for $m_{e} / m_{i}=0$. Since the growth rates are approximatively a factor two smaller for $m_{e} / m_{i}=0$ with respect to $\nu=0$, we conclude that InDWs are driving most of the SOL turbulent transport in the two plasma discharges considered here, in agreement with the conclusions in Ref. 12.

We note that, while $k_{Y}$ and $L_{n}$, input of the linear code, can be obtained from the nonlinear simulation results, they can also be estimated semi-analytically. In fact, assuming a negligible $\mathbf{E} \times \mathbf{B}$ shear flow and that the saturation of the growth of BMs and DWs is determined by the gradient removal mechanism, ${ }^{14}$ i.e., the saturation of the mode is due to the nonlinear local flattening of the plasma pressure profile, we obtain ${ }^{72}$

$$
L_{p}=\frac{L_{n}}{1+\eta}=\frac{q}{c_{s}}\left(\frac{\gamma}{k_{Y}}\right)_{\text {max }} .
$$

Equation (23) is an implicit equation for $L_{n}$ that is solved by scanning $\gamma$, solution of Eqs. (14)-(18), over the parameter space $\left(k_{Y}, L_{n}\right)$ and searching for the values of $L_{n}$ and $k_{Y}$ that satisfy Eq. (23). This procedure is applied to determine the equilibrium pressure gradient length of the two plasma discharges \#38373 and \#38413 for $m_{i} / m_{e}=800$, obtaining $L_{p}=44,56, k_{Y}=0.17,0.17$, and $\gamma=3.8,3.1$, respectively. The $L_{p}$ values computed according to Eq. (23) are in qualitative agreement with the results obtained from the nonlinear simulations discussed in Sec. III. Moreover, the poloidal wave number, $k_{Y}$, associated with the instability that drives most of the SOL turbulent transport is in good quantitative agreement with the nonlinear results.

Equation (23) allows us to investigate the impact of the reduced ion to electron mass ratio on our results. This is necessary since performing nonlinear simulations with $m_{i} /$ $m_{e}=3600$ is too demanding in terms of computational resources. Imposing a realistic ion to electron mass ratio $m_{i} /$ $m_{e}=3600$, Eq. (23) gives $L_{p}=39,52$ and $k_{Y}=0.14,0.16$ for the two considered plasma discharges. We see that $L_{p}$ and $k_{Y}$ are only slightly affected by increasing the ion to electron 
mass ratio to a realistic value. Using these $L_{p}$ and $k_{Y}$ as input parameters, we solve Eqs. (14)-(18) with $m_{i} / m_{e}=3600$, thus obtaining for the two considered discharges $\gamma=2.8$, $\gamma_{D W}=2.9$, and $\gamma_{B M}=0.1$. Moreover, solving Eqs. (19)-(22) for $\gamma$ with $L_{p}$ and $k_{Y}$ computed according to Eq. (23), we obtain $\gamma=4.6,3.7$ for $\nu=0$ and the realistic $m_{i} / m_{e}=3600$, while we have $\gamma=2.4,1.5$ for $m_{i} / m_{e}=0$. Therefore, we conclude that the same turbulent regime obtained with $m_{i} / m_{e}$ $=800$ is found also for the realistic $m_{i} / m_{e}=3600$, i.e., the SOL turbulent transport is mainly driven by $\mathrm{InDWs}$.

\section{CONCLUSIONS}

In the present paper, GBS simulations based on two RFX-mod plasma discharges with low edge safety factors are discussed, extending the work previously illustrated in Chapter 6 of Ref. 27. The GBS simulations are compared with experimental measurements, showing good qualitative and quantitative agreement for most of the considered quantities. Moreover, the SOL turbulent regime in the two discharges is identified.

The nonlinear simulations, carried out with GBS, are based on the two RFX-mod plasma discharges \#38373 and \#38413. In order to expand the GBS validation parameter regime and assess the reliability of the GBS model at low safety factor values, the simulation results are carefully compared with the experimental measurements. It is found that the numerical results are in good agreement with the experimental radial equilibrium profiles, fluctuation measurements, and higher order moments of $j_{s a t}$ and $V_{f l}$, except for the equilibrium profile of $V_{f l}$ and the level of fluctuations of $j_{\text {sat }}$. We infer that the observed discrepancies between simulations and experimental measurements are, at least in part, related to simulating only the tokamak SOL region, without including the plasma dynamics inside the LCFS, and to the limits of applicability of the drift reduced approximation.

For the two considered discharges, the simulation results indicate that the turbulent transport is mostly driven by DWs. To disentangle the effect of resistivity and electron inertia on the RFX-mod SOL dynamics, a linear model is considered. It is found that plasma adiabaticity is mostly broken by electron inertia, resulting in InDWs. Moreover, assuming that the linear growth of BMs and DWs saturates because of the nonlinear local flattening of the plasma pressure profile, the equilibrium pressure gradient length and the wave number associated with the instability that drives most of the turbulence transport are estimated with a quasi-linear theory, showing good agreement with the nonlinear results. This theory is then employed to investigate the impact of the reduced ion to electron mass that is used in the nonlinear simulations. It is found that InDWs are expected to drive the SOL turbulence also for the realistic $m_{i} / m_{e}=3600$.

\section{ACKNOWLEDGMENTS}

The simulations presented herein were carried out in part using the HELIOS supercomputer system at the Computational Simulation Centre of the International Fusion Energy Research Center (IFERC-CSC), Aomori, Japan, under the Broader Approach collaboration between Euratom and Japan, implemented by Fusion for Energy and JAEA; in part at the Swiss National Supercomputing Center (CSCS) under Projects ID s549, s655, and s718; and in part on the CINECA Marconi supercomputer within the framework of the GBSSOL project. This work has been carried out within the framework of the EUROfusion Consortium and has received funding from the Fond National Suisse de la Recherche Scientifique and from the Euratom research and training programme 2014-2018 under Grant Agreement No. 633053. The views and opinions expressed herein do not necessarily reflect those of the European Commission.

${ }^{1}$ A. Loarte, B. Lipschultz, A. S. Kukushkin, G. F. Matthews, P. C. Stangeby, N. Asakura, G. F. Counsell, G. Federici, A. Kallenbach, K. Krieger, A. Mahdavi, V. Philipps, D. Reiter, J. Roth, J. Strachan, D. Whyte, R. Doerner, T. Eich, W. Fundamenski, A. Herrmann, M. Fenstermacher, P. Ghendrih, M. Groth, A. Kirschner, S. Konoshima, B. LaBombard, P. Lang, A. W. Leonard, P. Monier-Garbet, R. Neu, H. Pacher, B. Pegourie, R. A. Pitts, S. Takamura, J. Terry, E. Tsitrone, and ITPA Scrape-off Layer and Divertor Physics Topical Group, "Chapter 4: Power and particle control," Nucl. Fusion 47, S203-S263 (2007).

${ }^{2}$ B. D. Dudson, M. V. Umansky, X. Q. Xu, P. B. Snyder, and H. R. Wilson, "BOUT++: A framework for parallel plasma fluid simulations," Comput. Phys. Commun. 180, 1467-1480 (2009).

${ }^{3}$ P. Ricci, F. D. Halpern, S. Jolliet, J. Loizu, A. Mosetto, A. Fasoli, I. Furno, and C. Theiler, "Simulation of plasma turbulence in scrape-off layer conditions: The GBS code, simulation results and code validation," Plasma Phys. Controlled Fusion 54, 124047 (2012).

${ }^{4}$ J. J. Rasmussen, A. H. Nielsen, J. Madsen, V. Naulin, and G. S. Xu, "Numerical modeling of the transition from low to high confinement in magnetically confined plasma," Plasma Phys. Controlled Fusion 58, 014031 (2016).

${ }^{5} \mathrm{P}$. Tamain, H. Bufferand, G. Ciraolo, C. Colin, P. Ghendrih, F. Schwander, and E. Serre, "3D properties of edge turbulent transport in full-torus simulations and their impact on poloidal asymmetries," Contrib. Plasma Phys. 54, 555-559 (2014).

${ }^{6}$ E. J. Doyle (Chair Transport Physics), W. A. Houlberg (Chair Confinement Database and Modelling), Y. Kamada (Chair Pedestal and Edge), V. Mukhovatov (co-Chair Transport Physics), T. H. Osborne (coChair Pedestal and Edge), A. Polevoi (co-Chair Confinement Database and Modelling), G. Bateman, J. W. Connor, J. G. Cordey (retired), T. Fujita, X. Garbet, T. S. Hahm, L. D. Horton, A. E. Hubbard, F. Imbeaux, F. Jenko, J. E. Kinsey, Y. Kishimoto, J. Li, T. C. Luce, Y. Martin, M. Ossipenko, V. Parail, A. Peeters, T. L. Rhodes, J. E. Rice, C. M. Roach, V. Rozhansky, F. Ryter, G. Saibene, R. Sartori, A. C. C. Sips, J. A. Snipes, M. Sugihara, E. J. Synakowski, H. Takenaga, T. Takizuka, K. Thomsen, M. R. Wade, H. R. Wilson, ITPA Transport Physics Topical Group, ITPA Confinement Database and Modelling Topical Group, and ITPA Pedestal and Edge Topical Group, "Chapter 2: Plasma confinement and transport," Nucl. Fusion 47, S18 (2007).

${ }^{7}$ R. Pitts, S. Carpentier, F. Escourbiac, T. Hirai, V. Komarov, A. Kukushkin, S. Lisgo, A. Loarte, M. Merola, R. Mitteau, A. Raffray, M. Shimada, and P. Stangeby, "Physics basis and design of the ITER plasmafacing components," J. Nucl. Mater. 415, S957-S964 (2011).

${ }^{8}$ G. Arnoux, T. Farley, C. Silva, S. Devaux, M. Firdaouss, D. Frigione, R. Goldston, J. Gunn, J. Horacek, S. Jachmich, P. Lomas, S. Marsen, G. Matthews, R. Pitts, M. Stamp, and P. Stangeby, "Scrape-off layer properties of ITER-like limiter start-up plasmas in JET," Nucl. Fusion 53 073016 (2013)

${ }^{9}$ A. Zeiler, D. Biskamp, J. F. Drake, and P. N. Guzdar, "Three-dimensional fluid simulations of tokamak edge turbulence," Phys. Plasmas 3, 2951-2960 (1996).

${ }^{10}$ B. D. Scott, "Drift wave versus interchange turbulence in tokamak geometry: Linear versus nonlinear mode structure," Phys. Plasmas 12, 062314 (2005).

${ }^{11}$ T. Rafiq, G. Bateman, A. H. Kritz, and A. Y. Pankin, "Development of drift-resistive-inertial ballooning transport model for tokamak edge plasmas," Phys. Plasmas 17, 082511 (2010).

${ }^{12}$ A. Mosetto, F. D. Halpern, S. Jolliet, J. Loizu, and P. Ricci, "Turbulent regimes in the tokamak scrape-off layer," Phys. Plasmas 20, 092308 (2013). 
${ }^{13}$ A. Mosetto, F. D. Halpern, S. Jolliet, and P. Ricci, "Low-frequency linearmode regimes in the tokamak scrape-off layer," Phys. Plasmas 19, 112103 (2012).

${ }^{14} \mathrm{P}$. Ricci and B. N. Rogers, "Plasma turbulence in the scrape-off layer of tokamak devices," Phys. Plasmas 20, 010702 (2013).

${ }^{15}$ O. Garcia, J. Horacek, R. Pitts, A. Nielsen, W. Fundamenski, V. Naulin, and J. J. Rasmussen, "Fluctuations and transport in the TCV scrape-off layer," Nucl. Fusion 47, 667-676 (2007).

${ }^{16}$ F. Militello, W. Fundamenski, V. Naulin, and A. H. Nielsen, "Simulations of edge and scrape off layer turbulence in mega ampere spherical tokamak plasmas," Plasma Phys. Controlled Fusion 54, 095011 (2012).

${ }^{17}$ N. Yan, A. H. Nielsen, G. S. Xu, V. Naulin, J. J. Rasmussen, J. Madsen, H. Q. Wang, S. C. Liu, W. Zhang, L. Wang, and B. N. Wan, "Statistical characterization of turbulence in the boundary plasma of EAST," Plasma Phys. Controlled Fusion 55, 115007 (2013).

${ }^{18}$ F. D. Halpern, J. L. Terry, S. J. Zweben, B. LaBombard, M. Podesta, and P. Ricci, "Comparison of 3D flux-driven scrape-off layer turbulence simulations with gas-puff imaging of Alcator C-Mod inner-wall limited discharges," Plasma Phys. Controlled Fusion 57, 054005 (2015).

${ }^{19}$ F. Nespoli, I. Furno, F. Halpern, B. Labit, J. Loizu, P. Ricci, and F. Riva, "Non-linear simulations of the TCV Scrape-Off Layer," Nucl. Mater. Energy 12, 1205-1208 (2016).

${ }^{20}$ R. Jorge, P. Ricci, F. D. Halpern, N. F. Loureiro, and C. Silva, "Plasma turbulence in the scrape-off layer of the ISTTOK tokamak," Phys. Plasmas 23, 102511 (2016).

${ }^{21}$ P. Tamain, H. Bufferand, G. Ciraolo, C. Colin, D. Galassi, P. Ghendrih, F. Schwander, and E. Serre, "The TOKAM3X code for edge turbulence fluid simulations of tokamak plasmas in versatile magnetic geometries," J. Comput. Phys. 321, 606-623 (2016).

${ }^{22}$ F. D. Halpern, P. Ricci, B. Labit, I. Furno, S. Jolliet, J. Loizu, A. Mosetto, G. Arnoux, J. P. Gunn, J. Horacek, M. Kočan, B. LaBombard, and C. Silva, "Theory-based scaling of the SOL width in circular limited tokamak plasmas," Nucl. Fusion 53, 122001 (2013).

${ }^{23}$ F. D. Halpern, P. Ricci, S. Jolliet, J. Loizu, and A. Mosetto, "Theory of the scrape-off layer width in inner-wall limited tokamak plasmas," Nucl. Fusion 54, 043003 (2014).

${ }^{24}$ F. D. Halpern, J. Horacek, R. A. Pitts, and P. Ricci, "A theoretical interpretation of the main scrape-off layer heat-flux width scaling for tokamak inner-wall limited plasmas," Plasma Phys. Controlled Fusion 58, 084003 (2016).

${ }^{25}$ P. Sonato, R. Piovan, and A. Luchetta, "Control of non-axisymmetric magnetic fields for plasma enhanced performances: The RFX contribution," Fusion Eng. Des. 74, 97-107 (2005).

${ }^{26}$ P. Martin, J. Adamek, P. Agostinetti, M. Agostini, A. Alfier, C. Angioni, V. Antoni, L. Apolloni, F. Auriemma, O. Barana, S. Barison, M. Baruzzo, P. Bettini, M. Boldrin, T. Bolzonella, D. Bonfiglio, F. Bonomo, A. Boozer, M. Brombin, J. Brotankova, A. Buffa, A. Canton, S. Cappello, L. Carraro, R. Cavazzana, M. Cavinato, L. Chacon, G. Chitarin, W. Cooper, S. D. Bello, M. Dalla Palma, R. Delogu, A. De Lorenzi, G. De Masi, J. Dong, M. Drevlak, D. Escande, F. Fantini, A. Fassina, F. Fellin, A. Ferro, S. Fiameni, A. Fiorentin, P. Franz, E. Gaio, X. Garbet, E. Gazza, L. Giudicotti, F. Gnesotto, M. Gobbin, L. Grando, S. Guo, Y. Hirano, S. Hirshman, S. Ide, V. Igochine, Y. In, P. Innocente, S. Kiyama, S. Liu, Y. Liu, D. Lòpez Bruna, R. Lorenzini, A. Luchetta, G. Manduchi, D. Mansfield, G. Marchiori, D. Marcuzzi, L. Marrelli, S. Martini, G. Matsunaga, E. Martines, G. Mazzitelli, K. McCollam, S. Menmuir, F. Milani, B. Momo, M. Moresco, S. Munaretto, L. Novello, M. Okabayashi, S. Ortolani, R. Paccagnella, R. Pasqualotto, M. Pavei, G. Perverezev, S. Peruzzo, R. Piovan, P. Piovesan, L. Piron, A. Pizzimenti, N. Pomaro, N. Pomphrey, I. Predebon, M. Puiatti, V. Rigato, A. Rizzolo, G. Rostagni, G. Rubinacci, A. Ruzzon, H. Sakakita, R. Sanchez, J. Sarff, F. Sattin, A. Scaggion, P. Scarin, W. Schneider, G. Serianni, P. Sonato, E. Spada, A. Soppelsa, S. Spagnolo, M. Spolaore, D. Spong, G. Spizzo, M. Takechi, C. Taliercio, D. Terranova, V. Toigo, M. Valisa, M. Veranda, N. Vianello, F. Villone, Z. Wang, R. White, D. Yadikin, P. Zaccaria, A. Zamengo, P. Zanca, B. Zaniol, L. Zanotto, E. Zilli, G. Zollino, and M. Zuin, "Overview of the RFX fusion science program,” Nucl. Fusion 51, 094023 (2011).

${ }^{27} \mathrm{~F}$. Riva, "Verification and validation procedures with applications to plasma-edge turbulence simulations," Ph.D. thesis (SB, Lausanne, 2017).

${ }^{28}$ P. Piovesan, D. Bonfiglio, F. Auriemma, F. Bonomo, L. Carraro, R. Cavazzana, G. De Masi, A. Fassina, P. Franz, M. Gobbin, L. Marrelli, P. Martin, E. Martines, B. Momo, L. Piron, M. Valisa, M. Veranda, N. Vianello, B. Zaniol, M. Agostini, M. Baruzzo, T. Bolzonella, A. Canton, S. Cappello, L. Chacón, G. Ciaccio, D. F. Escande, P. Innocente, R.
Lorenzini, R. Paccagnella, M. E. Puiatti, P. Scarin, A. Soppelsa, G. Spizzo, M. Spolaore, D. Terranova, P. Zanca, L. Zanotto, and M. Zuin, "RFX-mod: A multi-configuration fusion facility for three-dimensional physics studies," Phys. Plasmas 20, 056112 (2013).

${ }^{29}$ M. Spolaore, R. Cavazzana, L. Marrelli, L. Carraro, P. Franz, S. Spagnolo, B. Zaniol, M. Zuin, L. Cordaro, S. D. Bello, G. D. Masi, A. Ferro, C. Finotti, L. Grando, P. Innocente, O. Kudlacek, G. Marchiori, E. Martines, B. Momo, R. Paccagnella, P. Piovesan, C. Piron, M. E. Puiatti, M. Recchia, P. Scarin, C. Taliercio, N. Vianello, and L. Zanotto, "H-mode achievement and edge features in RFX-mod tokamak operation," in 26th IAEA Fusion Energy Conference, EX/P5-24. Kyoto, Japan (2016).

${ }^{30}$ M. Spolaore, N. Vianello, M. Agostini, R. Cavazzana, E. Martines, G. Serianni, P. Scarin, E. Spada, M. Zuin, and V. Antoni, "Magnetic and electrostatic structures measured in the edge region of the RFX-mod experiment," J. Nucl. Mater. 390-391, 448-451 (2009).

${ }^{31}$ N. Vianello, M. Spolaore, E. Martines, R. Cavazzana, G. Serianni, M. Zuin, E. Spada, and V. Antoni, "Drift-Alfvén vortex structures in the edge region of a fusion relevant plasma," Nucl. Fusion 50, 042002 (2010).

${ }^{32}$ H. Y. W. Tsui, R. D. Bengtson, G. X. Li, H. Lin, M. Meier, C. P. Ritz, and A. J. Wootton, "A new scheme for Langmuir probe measurement of transport and electron temperature fluctuations," Rev. Sci. Instrum. 63, 4608-4610 (1992)

${ }^{33}$ S. I. Braginskii, "Transport processes in a plasma," Rev. Plasma Phys. 1, 205 (1965).

${ }^{34}$ A. Zeiler, J. F. Drake, and B. Rogers, "Nonlinear reduced Braginskii equations with ion thermal dynamics in toroidal plasma," Phys. Plasmas 4 2134 (1997).

${ }^{35}$ F. D. Halpern, S. Jolliet, J. Loizu, A. Mosetto, and P. Ricci, "Ideal ballooning modes in the tokamak scrape-off layer," Phys. Plasmas 20, 052306 (2013).

${ }^{36}$ S. Jolliet, F. D. Halpern, J. Loizu, A. Mosetto, and P. Ricci, "Aspect ratio effects on limited scrape-off layer plasma turbulence," Phys. Plasmas 21 022303 (2014)

${ }^{37}$ A. Mosetto, F. D. Halpern, S. Jolliet, J. Loizu, and P. Ricci, "Finite ion temperature effects on scrape-off layer turbulence," Phys. Plasmas 22 , 012308 (2015).

${ }^{38}$ D. A. Russell, D. A. D'Ippolito, and J. R. Myra, "On relaxing the Boussinesq approximation in scrape-off layer turbulence (SOLT) model simulations," in Bulletin of the American Physical Society, Vol. 57, 54th Annual Meeting of the APS Division of Plasma Physics, BP8.159. Providence, Rhode Island, USA (2012).

${ }^{39}$ G. Q. Yu, S. I. Krasheninnikov, and P. N. Guzdar, "Two-dimensional modelling of blob dynamics in tokamak edge plasmas," Phys. Plasmas 13, 042508 (2006).

${ }^{40}$ K. Bodi, G. Ciraolo, P. Ghendrih, F. Schwander, E. Serre, and P. Tamain, "Impact of the Boussinesq approximation in tokamak scrape-off layer turbulence," in 38th EPS Conference on Plasma Physics, P1.121. Strasbourg, France (2011).

${ }^{41}$ F. D. Halpern, P. Ricci, S. Jolliet, J. Loizu, J. Morales, A. Mosetto, F. Musil, F. Riva, T. M. Tran, and C. Wersal, "The GBS code for tokamak scrape-off layer simulations," J. Comput. Phys. 315, 388-408 (2016).

${ }^{42}$ B. N. Rogers and P. Ricci, "Low-frequency turbulence in a linear magnetized plasma," Phys. Rev. Lett. 104, 225002 (2010).

${ }^{43}$ P. Ricci, B. N. Rogers, and S. Brunner, "High- and low-confinement modes in simple magnetized toroidal plasmas," Phys. Rev. Lett. 100, 225002 (2008).

${ }^{44} \mathrm{P}$. Ricci and B. N. Rogers, "Transport scaling in interchange-driven toroidal plasmas," Phys. Plasmas 16, 062303 (2009).

${ }^{45} \mathrm{P}$. Ricci and B. N. Rogers, "Turbulence phase space in simple magnetized toroidal plasmas," Phys. Rev. Lett. 104, 145001 (2010).

${ }^{46}$ J. Loizu, P. Ricci, F. D. Halpern, and S. Jolliet, "Boundary conditions for plasma fluid models at the magnetic presheath entrance," Phys. Plasmas 19, 122307 (2012).

${ }^{47}$ A. Arakawa, "Computational design for long-term numerical integration of the equations of fluid motion: Two-dimensional incompressible flow. Part I," J. Comput. Phys. 1, 119-143 (1966).

${ }^{48} \mathrm{~A}$. Mosetto, "Turbulent regimes in the tokamak scrape-off layer," $\mathrm{Ph} . \mathrm{D}$ thesis (SB, Lausanne, 2014).

${ }^{49}$ O. E. Garcia, J. Horacek, R. A. Pitts, A. H. Nielsen, W. Fundamenski, J. P. Graves, V. Naulin, and J. J. Rasmussen, "Interchange turbulence in the TCV scrape-off layer," Plasma Phys. Controlled Fusion 48, L1-L10 (2006).

${ }^{50}$ D. A. D’Ippolito, J. R. Myra, and S. J. Zweben, "Convective transport by intermittent blob-filaments: Comparison of theory and experiment," Phys. Plasmas 18, 060501 (2011). 
${ }^{51}$ D. Carralero, P. Manz, L. Aho-Mantila, G. Birkenmeier, M. Brix, M. Groth, H. W. Müller, U. Stroth, N. Vianello, and E. Wolfrum, "Experimental validation of a filament transport model in turbulent magnetized plasmas," Phys. Rev. Lett. 115, 215002 (2015).

${ }^{52}$ F. Halpern and P. Ricci, "Velocity shear, turbulent saturation, and steep plasma gradients in the scrape-off layer of inner-wall limited tokamaks," Nucl. Fusion 57, 034001 (2017).

${ }^{53}$ F. Riva, P. Ricci, F. D. Halpern, S. Jolliet, J. Loizu, and A. Mosetto, "Verification methodology for plasma simulations and application to a scrape-off layer turbulence code," Phys. Plasmas 21, 062301 (2014).

${ }^{54}$ C. K. Tsui, J. A. Boedo, F. D. Halpern, J. Loizu, F. Nespoli, J. Horacek, B. Labit, J. Morales, H. Reimerdes, P. Ricci, C. Theiler, S. Coda, B. P. Duval, and I. Furno, "Poloidal asymmetry in the narrow heat flux feature in the TCV scrape-off layer," Phys. Plasmas 24, 062508 (2017).

${ }^{55}$ E. Sánchez, C. Hidalgo, D. López-Bruna, I. García-Cortés, R. Balbín, M. A. Pedrosa, B. van Milligen, C. Riccardi, G. Chiodini, J. Bleuel, M. Endler, B. A. Carreras, and D. E. Newman, "Statistical characterization of fluctuation wave forms in the boundary region of fusion and nonfusion plasmas," Phys. Plasmas 7, 1408-1416 (2000).

${ }^{56}$ Y. H. Xu, S. Jachmich, R. R. Weynants, and TEXTOR Team, "On the properties of turbulence intermittency in the boundary of the TEXTOR tokamak," Plasma Phys. Controlled Fusion 47, 1841-1855 (2005).

${ }^{57}$ I. Nanobashvili, J. Gunn, and P. Devynck, "Radial profiles of plasma turbulent fluctuations in the scrape-off layer of the Tore Supra tokamak," J. Nucl. Mater. 363-365, 622-627 (2007).

${ }^{58}$ P. Ricci, C. Theiler, A. Fasoli, I. Furno, B. Labit, S. H. Muller, M. Podesta, and F. M. Poli, "Langmuir probe-based observables for plasmaturbulence code validation and application to the TORPEX basic plasma physics experiment," Phys. Plasmas 16, 055703 (2009).

${ }^{59}$ H. Wang, G. Xu, H. Guo, B. Wan, V. Naulin, S. Ding, N. Yan, W. Zhang, L. Wang, S. Liu, R. Chen, L. Shao, H. Xiong, P. Liu, M. Jiang, and G.-N. Luo, "Observation of a new turbulence-driven limit-cycle state in $\mathrm{H}$ modes with lower hybrid current drive and lithium-wall conditioning in the EAST superconducting tokamak," Nucl. Fusion 52, 123011 (2012).

${ }^{60}$ C. P. Ritz, E. J. Powers, T. L. Rhodes, R. D. Bengtson, K. W. Gentle, H. Lin, P. E. Phillips, A. J. Wootton, D. L. Brower, N. C. Luhmann, W. A. Peebles, P. M. Schoch, and R. L. Hickok, "Advanced plasma fluctuation analysis techniques and their impact on fusion research (invited)," Rev. Sci. Instrum. 59, 1739-1744 (1988).

${ }^{61}$ C. A. F. Varandas, J. A. C. Cabral, J. T. Mendonça, M. P. Alonso, P. Amorim, B. B. Carvalho, C. Correia, L. Cupido, M. L. Carvalho, J. M. Dias, H. Fernandes, C. J. Freitas, S. Magalhães, A. Malaquias, M. E. Manso, A. Praxedes, J. Santana, F. Serra, A. Silva, A. Soares, J. Sousa, W. van Toledo, P. Vaessen, P. Varela, S. Vergamota, and B. de Groot, "Engineering aspects of the tokamak ISTTOK," Fusion Technol. 29, 105-115 (1996).

${ }^{62}$ T. T. Ribeiro and B. Scott, "Gyrofluid turbulence studies of the effect of the poloidal position of an axisymmetric Debye sheath," Plasma Phys. Controlled Fusion 50, 055007 (2008).

${ }^{63}$ J. W. Connor, R. J. Hastie, and J. B. Taylor, "Shear, periodicity, and plasma ballooning modes," Phys. Rev. Lett. 40, 396-399 (1978).

${ }^{64}$ G. Bateman and D. B. Nelson, "Resistive-ballooning-mode equation," Phys. Rev. Lett. 41, 1804-1807 (1978).

${ }^{65}$ D. R. McCarthy, P. N. Guzdar, J. F. Drake, T. M. Antonsen, and A. B. Hassam, "Stability of resistive and ideal ballooning modes in the Texas Experimental Tokamak and DIII-D,” Phys. Fluids B: Plasma Phys. 4, 1846 (1992).

${ }^{66}$ S. V. Novakovskii, P. N. Guzdar, J. F. Drake, C. S. Liu, and F. L. Waelbroeck, "New unstable branch of drift resistive ballooning modes in tokamaks," Phys. Plasmas 2, 781 (1995).

${ }^{67}$ K. Mima and A. Hasegawa, "Nonlinear instability of electromagnetic drift waves," Phys. Fluids 21, 81 (1978).

${ }^{68} \mathrm{M}$. Wakatani and A. Hasegawa, "A collisional drift wave description of plasma edge turbulence,” Phys. Fluids 27, 611 (1984).

${ }^{69} \mathrm{H}$. Sugama, M. Wakatani, and A. Hasegawa, "Study of resistive drift and resistive interchange modes in a cylindrical plasma with magnetic shear," Phys. Fluids 31, 1601 (1988).

${ }^{70}$ W. Horton, "Drift waves and transport," Rev. Mod. Phys. 71, 735-778 (1999).

${ }^{71}$ P. H. Diamond, A. Hasegawa, and K. Mima, "Vorticity dynamics, drift wave turbulence, and zonal flows: A look back and a look ahead," Plasma Phys. Controlled Fusion 53, 124001 (2011).

${ }^{72}$ F. Riva, E. Lanti, S. Jolliet, and P. Ricci, "Plasma shaping effects on tokamak scrape-off layer turbulence," Plasma Phys. Controlled Fusion 59, 035001 (2017). 